\title{
Parameterized Problems Complete for Nondeterministic FPT time and Logarithmic Space*
}

\author{
Hans L. Bodlaender ${ }^{\dagger}$, Carla Groenland ${ }^{1}$, Jesper Nederlof ${ }^{1}$, and \\ Céline M. F. Swennenhuis ${ }^{2}$ \\ ${ }^{1}$ Department of Information and Computing Sciences, Utrecht \\ University, the Netherlands \\ ${ }^{2}$ Department of Mathematics and Computer Science, Eindhoven \\ University of Technology, The Netherlands
}

\begin{abstract}
Let XNLP be the class of parameterized problems such that an instance of size $n$ with parameter $k$ can be solved nondeterministically in time $f(k) n^{O(1)}$ and space $f(k) \log (n)$ (for some computable function $f)$. We give a wide variety of XNLP-complete problems, such as LIST Coloring and Precoloring Extension with pathwidth as parameter, Scheduling of Jobs with Precedence Constraints, with both number of machines and partial order width as parameter, BANDWIDTH and variants of WEIGHTED CNF-SATISFIABILITY and reconfiguration problems. In particular, this implies that all these problems are $W[t]$-hard for all $t$. This also answers a long standing question on the parameterized complexity of the BANDWIDTH problem.
\end{abstract}

\section{Introduction}

Already since the 1970's, an important paradigm in classical complexity theory has been that an increased number of alternations of existential and universal quantifiers increases the complexity of search problems: This led to the central definition of the polynomial hierarchy [42, whose study resulted in cornerstone results in complexity theory such as Toda's theorem and lower bounds for

${ }^{*}$ The work of the second and third author was supported by the project CRACKNP that has received funding from the European Research Council (ERC) under the European Union's Horizon 2020 research and innovation programme (grant agreement No 853234). The work of the fourth author was supported by the Netherlands Organization for Scientific Research under project no. 613.009.031b.

${ }^{\dagger}$ Corresponding author. Email: h.l.bodlaender@uu.nl 
time/space tradeoffs for SAT [2]. In their foundational work in the early 1990s, Downey and Fellows introduced an analogue of this hierarchy for parameterized complexity theory, called the $W$-hierarchy. This hierarchy comprises of the complexity classes FPT, the parameterized analogue of $P, W[1]$, the parameterized analogue of $N P$, and the classes $W[2], \ldots, W[P], X P$ (see e.g. [15, 16, 17]).

While in the polynomial hierarchy only the classes with no quantifier alternation (i.e. $P, N P$ and $c o-N P$ ) are prominent, many natural parameterized problems are known to be hard or even complete for $W[i]$ for some $i>1$. Thus, the $W$-hierarchy substantially differentiates the complexity of hard parameterized problems. And such a differentiation has applications outside parameterized complexity as well: For example, for problems in $W[1]$ we can typically improve over brute-force enumeration algorithms, while for problems in $W[2]$ we can prove lower bounds under the Strong Exponential Time Hypothesis excluding such improvements (see e.g. the discussion in [1]) 1

For several problems, completeness for a class is known, e.g., CLIQUE is $W[1]$ complete [16] and Dominating Set is $W[2]$-complete [15]. However, there are also several problems known to be hard for $W[1], W[2]$, or even for $W[t]$ for all positive integers $t$, but which are not known to be in the class $W[P]$; in many cases, only membership in XP was known. For such problems, it is an intriguing question to establish their exact position within the $W$-hierarchy as it can be expected to shed light on their complexity similarly as it did for the previous problems.

One example of such a problem is the BANDwIDTH problem. It is known to be hard for all classes $W[t]$ since 1994[9], with a recently published proof that this already holds for a special subclass of trees (namely, caterpillars with hair length at most three [4). However, already in the midst of the 1990s, Hallett argued that it is unlikely that BANDWIDTH belongs to $W[P]$, see the discussion by Fellows and Rosamond in 24. The argument intuitively boils down to the following: BANDWIDTH 'seems' to need certificates with $\Omega(n)$ bits, while problems in $W[P]$ have certificates with $O(f(k) \log n)$ bits. A similar situation applies to several other $W[1]$-hard problems.

A (largely overlooked) breakthrough was made a few years ago by Elberfeld et al. 21], who studied several classes of parameterized problems, including a class which they called $N[$ fpoly,$f \log ]$ : parameterized problems that can be solved with a non-deterministic algorithm with simultaneously, the running time bounded by $f(k) n^{c}$ and the space usage bounded by $f(k) \log n$, with $k$ the parameter, $n$ the input size, $c$ a constant, and $f$ a computable function. For easier future reference, we denote this class by XNLP. Elberfeld et al. 21] showed that a number of problems are complete for this class, including the LONGEST Common Substring problem. Since 1995, Longest Common Substring is known to be hard for all $W[t][6]$, but its precise parameterized complexity was unknown until the result by Elberfeld et al. 21.

\footnotetext{
${ }^{1}$ For example the naïve algorithm $O\left(n^{k+1}\right)$ time algorithm for finding cliques on $k$ vertices on $n$-vertex graphs can be improved to run in $n^{0.8 k}$ time, but similar run times for DominATiNG SET refute the Strong Exponential Time Hypothesis.
} 
Our contribution We show that the class XNLP (i.e., $N[$ fpoly, $f \log ]$ ) can play an important role in establishing the parameterized complexity of a large collection of well studied problems, ranging from abstract problems on different types of automata (see e.g. [21] or later in this paper), logic, graph theory, scheduling, and more. In this paper, we give a number of different examples of problems that are complete for XNLP. These include BANDwIDTH, thus indirectly answering a question that was posed over 25 years ago.

\begin{tabular}{|c|c|}
\hline Problem & Source \\
\hline LONGEST COMMON SUBSEQUENCE + & 21 \\
\hline Timed Non-Determ. Cellular Automaton + & [21], see Subsection 2.5] \\
\hline Chained CNF-Satisfiability + & Subsection 3.1 \\
\hline Chained Multicolored Clique & Subsection 3.2 \\
\hline ACCEPTING NNCCM & Subsection 3.3 \\
\hline LisT COLORING pw + & Subsection 4.1 \\
\hline LOG-PATHWIDTH DS, IS & Subsection 4.2 \\
\hline SCHEDULING WITH PRECEDENCE CONSTAINTS & Subsection $\overline{4.3}$ \\
\hline Uniform Emulation of Weighted Paths & Subsection 4.4 \\
\hline BANDWIDTH & Subsection 4.5 (with [4) \\
\hline Timed DS (IS, CL) Reconfiguration & Subsection 4.6 \\
\hline ACYClic Finite State Automata Intersection & [46]; Appendix A \\
\hline
\end{tabular}

Table 1: An overview of XNLP-complete problems is given. For problems marked with + , the source also gives an XNLP-hardness or completeness proofs for variants of the stated problem. We use the abbreviations CL = Clique, IS $=$ Independent Set, DS = Dominating Set, $\mathrm{pw}=$ parameterized by pathwidth.

In Table 1. we list the problems shown to be XNLP-complete in either this paper or by Elberfeld et al. 21]. Figure 1 shows for the problems from which problem the reduction starts to show XNLP-hardness.

Often, membership in XNLP can be seen by looking at the algorithm that establishes membership in XP. Many problems in XNLP typically have a dynamic programming algorithm that sequentially builds tables, with each individual table entry expressible with $O(f(k) \log n)$ bits. We then get membership in XNLP by instead of tabulating all entries of a table, guessing one entry of the next table - the step resembles the text-book transformation between a deterministic and non-deterministic finite automaton.

Interestingly, hardness for the class XNLP also has consequences for the use of memory of deterministic parameterized algorithms. Pilipczuk and Wrochna 39] conjecture that Longest Common Subsequence (variant 1) has no XP algorithm that runs in $f(k) n^{c}$ space, for a computable function $f$ and constant $c$; if this conjecture holds, then no XNLP-hard problem has such an algorithm. See Section 5 for more details.

When a problem is XNLP-hard, it is also hard for each class $W[t]$ (see Lemma 2.2. Thus, XNLP-hardness proofs are also a tool to show hardness for $W[t]$ for all $t$. In this sense, our results strengthen existing results from the 


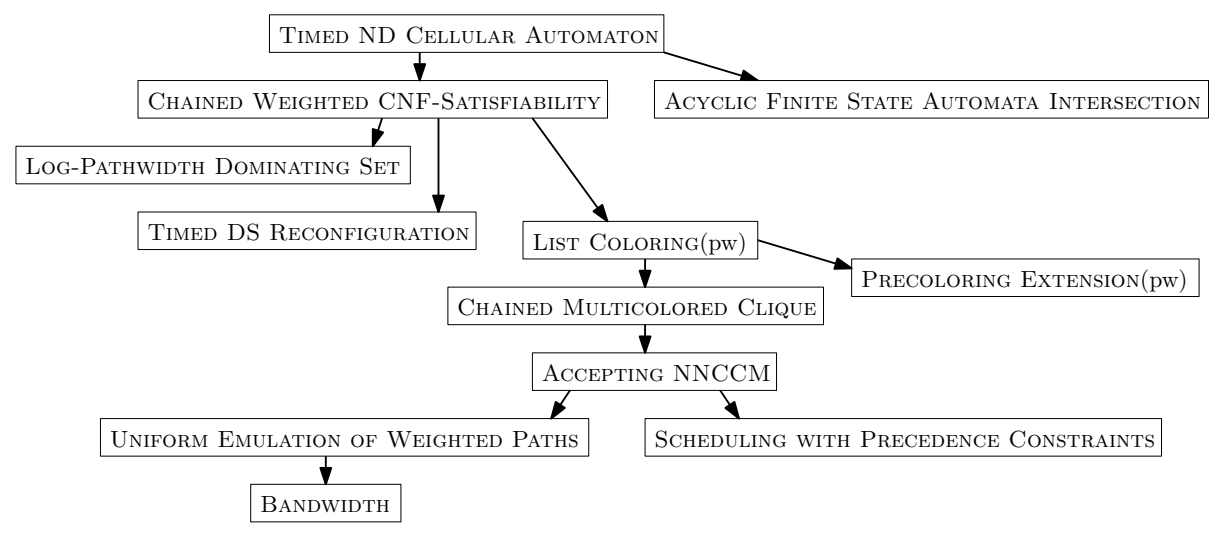

Figure 1: Reductions between XNLP-hard problems from this paper. Several variants of problems are not shown.

literature: for example, List Coloring and Precoloring Extension parameterized by pathwidth (or treewidth) were known to be $W[1]$-hard [22, and Precedence Constraint $K$-Processor Scheduling parameterized by the number of processors $K$ was known to be $W[2]$-hard [8]. Our XNLP-hardness proofs imply hardness for $W[t]$ for all $t$. Moreover, our XNLP-hardness proofs are often simpler than the existing proofs that problems are hard for $W[t]$ for all $t$.

Related to the class XNLP is the class XNL: the parameterized problems that can be solved by a nondeterministic algorithm that uses $f(k) \log n$ space. There is no explicit time bound, but we can freely add a time bound of $2^{f(k) \log n}$, and thus XNL is a subset of XP. XNL can be seen as the parameterized counterpart of NL. Amongst others, XNL was studied by Chen et al. [13], who showed that Compact Turing Machine Computation is complete for XNL.

Hardness for a class is always defined with respect to a class of reductions. In our proofs, we use parameterized logspace reductions (or, in short, pl-reductions). A brief discussion of other reductions can be found in Subsection 5.2

Paper overview. In Section 2, we give a number of preliminary definitions and results. In Section 3 we introduce three new problems and prove that they are XNLP-complete. In Section 4 we then use these problems as building blocks, to prove other problems to be either XNLP-complete or XNLP hard. For each of the problems, its background and a short literature review specific to it will be given inside its relevant subsection. Some related variants have been moved to the appendix. Final comments and open problems are given in Section 5 . 


\section{Preliminaries}

In this section we formally define the class XNLP and give some preliminary results.

The section is organized as follows: first we introduce some basic notions in Subsection 2.1. next we formally define the class XNLP in Subsection 2.2. In Subsection 2.3 we then introduce the type of reductions that will be used in this paper and in Subsection 2.4 we go over some preliminary results. Subsection 2.5 ends the section with a discussion of Cellular automata, for which Elberfeld et al. 21 already established it was XNLP complete. From this problem we will (indirectly) derive the XNLP-hardness for all other XNLP-hard problems in this paper.

\subsection{Basic notions}

We assume the reader to be familiar with a number of notions from complexity theory, parameterized algorithms, and graph theory. A few of these are reviewed below, along with some new and less well-known notions.

We use interval notation for sets of integers, i.e., $[a, b]=\{i \in \mathbb{Z} \mid a \leq i \leq b\}$. All logarithms in this paper have base 2. $\mathbf{N}$ denotes the set of the natural numbers $\{0,1,2, \ldots\}$, and $\mathbf{Z}^{+}$denotes the set of the positive natural numbers $\{1,2, \ldots\}$.

\subsection{Definition of the class XNLP}

In this paper, we study parameterized decision problems, which are subsets of $\Sigma^{*} \times \mathbf{N}$, for a finite alphabet $\Sigma$. The following notation is used, also by e.g. 21, to denote classes of (non-)deterministic parameterized decision problems with a bound on the used time and space. Here, we use the following notations: poly for a polynomial function in the input size; $\log$ for $O(\log n) ; n$ for the input size; $f$ for a computable function of the parameter; $\infty$ if there is no a priory bound for the resource. Let $D[t, s]$ denote the class of parameterized decision problems that can be solved by a deterministic algorithm in $t$ time and $s$ space and let $N[t, s]$ be analogously defined for non-deterministic algorithms. Thus, FPT can be denoted by $D[$ fpoly, $\infty]$; we can denote XP by $D\left[n^{f}, \infty\right]$, NP by $N[$ poly,$\infty], L$ by $D[\infty, \log ]$, etcetera.

A special role in this paper is played by the class $N[f$ poly,$f \log ]$ : the parameterized decision problems that can be solved by a non-deterministic algorithm that simultaneously uses at most $f(k) n^{c}$ time and at most $f(k) \log n$ space, on an input $(x, k)$, where $x$ can be denoted with $n$ bits, $f$ a computable function, and $c$ a constant. Because of the special role of this class, we use the shorter notation XNLP.

XNLP is a subclass of the class XNL, which was studied by Chen et al. 13. $\mathrm{XNL}$ is the class of problems solvable with a non-deterministic algorithm in $f(k) \log n$ space $(f, k, n$ as above), i.e, XNL is the class $N[\infty, f \log ]$. 
We assume that the reader to be familiar with notions from parameterized complexity, such as XP, $W[1], W[2], \ldots, W[P]$ (see e.g. [17]). For classes of parameterized problems, we can often make a distinction between non-uniform (a separate algorithm for each parameter value), and uniform. Throughout this paper, we look at the uniform variant of the classes, but we also will assume that functions $f$ of the parameter in time and resource bounds are computable — this is called strongly uniform by Downey and Fellows [17].

\section{$2.3 \quad$ Reductions}

Hardness for a class is defined in terms of reductions. We mainly use parameterized logspace reductions, which are a special case of fixed parameter tractable reductions. Both are defined below; the definitions are based upon the formulations in [21]. Two other types of reductions are briefly discussed in the Conclusion (Section 5.2.)

- A parameterized reduction from a parameterized problem $Q_{1} \subseteq \Sigma_{1}^{*} \times \mathbf{N}$ to a parameterized problem $Q_{2} \subseteq \Sigma_{2}^{*} \times \mathbf{N}$ is a function $f: \Sigma_{1}^{*} \times \mathbf{N} \rightarrow \Sigma_{2}^{*} \times \mathbf{N}$, such that the following holds.

1. For all $(x, k) \in \Sigma_{1}^{*} \times \mathbf{N},(x, k) \in Q_{1}$ if and only if $f((x, k)) \in Q_{2}$.

2. There is a computable function $g$, such that for all $(x, k) \in \Sigma_{1}^{*} \times \mathbf{N}$, if $f((x, k))=\left(y, k^{\prime}\right)$, then $k^{\prime} \leq g(k)$.

- A parameterized logspace reduction or pl-reduction is a parameterized reduction for which there is an algorithm that computes $f((x, k))$ in space $O(g(k)+\log n)$, with $g$ a computable function and $n=|x|$ the number of bits to denote $x$.

- A fixed parameter tractable reduction or fpt-reduction is a parameterized reduction for which there is an algorithm that computes $f((x, k))$ in time $O\left(g(k) n^{c}\right)$, with $g$ a computable function, $n=|x|$ the number of bits to denote $x$ and $c$ a constant.

In the remainder of the paper, unless stated otherwise, completeness for $\mathrm{XNLP}$ is with respect to pl-reductions.

\subsection{Preliminary results on XNLP}

We give some easy observations that relate XNLP to other notions from parameterized complexity. The following easy observation can be seen as a special case of the fact that $N[\infty, S(n)] \subseteq D\left[2^{S(n)}, \infty\right]$, see [2, Theorem 4.3].

Lemma 2.1. $X N L P$ is a subset of $X P$.

Proof. Using standard techniques, we can transform the non-deterministic algorithm to a deterministic algorithm that employs dynamic programming: tab-

ulate all reachable configurations of the machine (a configuration is a tuple, 
consisting of the contents of the work tape, the state of the machine, and the position of the two headers). From a configuration, we can compute all configurations that can be reached in one step, and thus we can check if a configuration that has an accepting state can be reached.

The number of such configurations is bounded by the product of a single exponential of the size of the work tape (i.e., at most $2^{f(k) \log n}=n^{f(k)}$ for some computable function $f$ ), the constant number of states of the machine, and the $O(f(k) \log n) \cdot n$ number of possible pairs of locations of the heads, and thus bounded by a function of the form $n^{g(k)}$ with $g$ a computable function.

Lemma 2.2. If a parameterized problem $Q$ is XNLP-hard, then it is hard for each class $W[t]$ for all $t \in \mathbf{Z}^{+}$.

Proof. Observe that the $W[t]$-complete problem Weighted $t$-Normalized Satisfiability belongs to XNLP. (In Weighted $t$-Normalized SatisfiaBILITY, we have a Boolean formula with parenthesis-depth $t$ and ask if we can satisfy it by setting exactly $k$ variables to true and all others to false; we can non-deterministically guess which of the $k$ Boolean variables are true; verifying whether this setting satisfies the formula can be done with $O(t+k \log n)$ bits of space, see e.g. [17.)

Each problem in $W[t]$ has an fpt-reduction to Weighted $t$-Normalized SATisfiability, and the latter has a pl-reduction (which is also an fpt-reduction) to any XNLP-hard problem $Q$. The transitivity of fpt-reductions implies that $Q$ is then also hard for $W[t]$.

Lemma 2.3 (Chen et al. [13]). If $N L \neq P$, then there are parameterized problems in FPT that do not belong to XNL (and hence also not to XNLP).

Proof. Take a problem $Q$ that belongs to P, but not to NL. Consider the parameterized problem $Q^{\prime}$ with $(x, k) \in Q^{\prime}$ if and only if $x \in Q$. (We just ignore the parameter part of the input.) Then $Q^{\prime}$ belongs to FPT, since the polynomial time algorithm for $Q$ also solves $Q^{\prime}$. If $Q^{\prime}$ is in XNL, then there is an algorithm that solves $Q$ in (non-deterministic) logarithmic space, a contradiction. So $Q^{\prime}$ belongs to FPT but not to XNL.

Chen et al. [13] introduce the following problem.

CNTMC

Input: the encoding of a non-deterministic Turing Machine $M$; the encoding of a string $x$ over the alphabet of the machine.

Parameter: $k$.

Question: Is there an accepting computation of $M$ on input $x$ that visits at most $k$ cells of the work tape?

Theorem 2.4 (Chen et al. [13]). CNTMC is XNL-complete under pl-reductions.

It is possible to show XNLP-completeness for a 'timed' variant of this problem. 


\section{TIMED CNTMC}

Input: the encoding of a non-deterministic Turing Machine $M$; the encoding of a string $x$ over the alphabet of the machine; an integer $T$ given in unary.

Parameter: $k$.

Question: Is there an accepting computation of $M$ on input $x$ that visits at most $k$ cells of the work tape and uses at most $T$ time?

The fact that the time that the machine uses is given in unary, is needed to show membership in XNLP.

Theorem 2.5. TIMED CNTMC is XNLP-complete.

We state the result without proof, as the proof is similar to the proof of Theorem 2.4 from [13, and we do not build upon the result. We instead start with a problem on cellular automata which was shown to be complete for XNLP by Elberfeld et al. 21. We discuss this problem in the next subsection. Elberfeld et al. 21] show a number of other problems to be XNLP-complete, including a timed version of the acceptance of multihead automata, and the LONGEST Common Subsequence problem, parameterized by the number of strings. The latter result is discussed in the Conclusion, Section 5.1.

\subsection{Cellular automata}

In this subsection, we discuss one of the results by Elberfeld et al. 21. Amongst the problems that are shown to be complete for XNLP by Elberfeld et al. 21, of central importance to us is the Timed Non-Deterministic Cellular AuTOMATON problem. We use the hardness of this problem to show the hardness of Chained CNF-Satisfiability in Subsection 3.1 .

In this subsection, we describe the Timed Non-Deterministic Cellular Automaton problem, and a variant. We are given a linear cellular automaton, a time bound $t$ given in unary, and a starting configuration for the automaton, and ask if after $t$ time steps, at least one cell is in an accepting state.

More precisely, we have a set of states $S$. We assume there are two special states $s_{L}$ and $s_{R}$ which are used for the leftmost and rightmost cell. A configuration is a function $c:\{1, \ldots, q\} \rightarrow S$, with $c(1)=s_{L}, c(q)=s_{R}$ and for $i \in[2, q-1], c(i) \in S \backslash\left\{s_{L}, s_{R}\right\}$. We say that we have $q$ cells, and in configuration $c$, cell $i$ has state $c(i)$. The machine is further described by a collection of 4-tuples $\mathcal{T}$ in $S \times\left(S \backslash\left\{s_{L}, s_{R}\right\}\right) \times S \times\left(S \backslash\left\{s_{L}, s_{R}\right\}\right)$. At each time step, each cell $i \in[2, q]$ reads the 3 -tuple $\left(s_{1}, s_{2}, s_{3}\right)$ of states given by the current states of the cells $i-1, i$ and $i+1$ (in that order). If there is no 4 -tuple of the form $\left(s_{1}, s_{2}, s_{3}, s_{4}\right)$ for some $s_{4} \in S$, then the machine halts and rejects; otherwise, the cell selects an $s_{4} \in S$ with $\left(s_{1}, s_{2}, s_{3}, s_{4}\right) \in \mathcal{T}$ and moves in this time step to state $s_{4}$. (In a non-deterministic machine, there can be multiple such states $s_{4}$ and a non-deterministic step is done. For a deterministic cellular automaton, for each 3 -tuple $\left(s_{1}, s_{2}, s_{3}\right)$ there is at most one 4 -tuple $\left(s_{1}, s_{2}, s_{3}, s_{4}\right) \in \mathcal{T}$.) Note that the leftmost and rightmost cell never change state: their states are used to mark the ends of the tape of the automaton. 


\section{Timed Non-Deterministic Cellular Automaton}

Input: Cellular automaton with set of states $S$ and set of transitions $\mathcal{T}$; configuration $c$ on $q$ cells; integer in unary $t$; subset $A \subseteq S$ of accepting states.

Parameter: $q$.

Question: Is there an execution of the machine for exactly $t$ steps with initial configuration $c$, such that at time $t$ at least one cell of the automaton is in $A$ ?

We will build on the following result.

Theorem 2.6 (Elberfeld et al. 21]). Timed Non-Deterministic Cellular Automaton is XNLP-complete.

We recall that the class, denoted by XNLP in the current paper, is called $N[$ fpoly, $f \log ]$ in [21].

Elberfeld et al. 21] state that asking that all cells are in an accepting state does not make a difference, i.e., if we modify the TIMED Non-DETERMINISTIC Cellular Automaton problem by asking if all cells are in an accepting state at time $t$, then we also have an XNLP-complete problem.

We also discuss a variant that can possibly be useful as another starting point for reductions.

Timed Non-halting Non-deterministic Cellular AutomaTON

Input: Cellular automaton with set of states $S$ and set of transitions $\mathcal{T}$; configuration $c$ on $q$ cells; integer in unary $t$; subset $A \subseteq S$ of accepting states.

Parameter: $q$.

Question: Is there an execution of the machine for exactly $t$ steps with initial configuration $c$, such that the machine does not halt before time $t$ ?

Corollary 2.7. Timed Non-halting Non-deterministic Cellular AuTOMATON is XNLP-complete.

Proof. Membership in XNLP follows in the same way as for Timed Nondeterministic Cellular Automaton, see 21]; observe that we can store a configuration using $\lceil q \log |S|\rceil$ bits.

Hardness follows by modifying the automaton as follows. We take an automaton that accepts, if and only if at time $t$ all cells are in an accepting state. Now, we enlarge the set of states as follows: for each time step $t^{\prime} \in[0, t]$, and each state $s \in S \backslash\left\{s_{L}, s_{R}\right\}$, we create a state $s^{t^{\prime}}$. The initial configuration $c$ is modified to $c^{\prime}$ by setting $c^{\prime}(i)=s^{0}$ for $i \in[2, q-1]$ when $c(i)=s$. We enlarge the set of transitions as follows. For each $t^{\prime} \in[0, t-1]$ and $\left(s_{1}, s_{2}, s_{3}, s_{4}\right) \in \mathcal{T}$, we create a transition $\left(s_{1}^{t^{\prime}}, s_{2}^{t^{\prime}}, s_{3}^{t^{\prime}}, s_{4}^{t^{\prime}+1}\right)$ in the new set of transitions. In this way, each state of the machine also codes the time: at time $t^{\prime}$ all cells except the first and last will have a state of the form $s^{t^{\prime}}$. 
We run the machine for one additional step, i.e., we increase $t$ by one. We create one additional accepting state $s_{a}$. For each accepting state $s \in A$, we make transitions $\left(x, s^{t}, y, s_{a}\right)$ for all possible values $x$ and $y$ can take. When $s \notin A$, then there are no $x, y, z$ for which there is a transition of the form $\left(x, s^{t}, y, z\right)$. This ensures that a cell has a possible transition at time $t$ if and only if it is in an accepting state. In particular, when all states are accepting, all cells have a possible transition at time $t$; if there is a state that is not accepting at time $t$, then the machine halts.

\section{Building Blocks}

In this section, we introduce three new problems and prove that they are XNLPcomplete, namely Chained CNF-Satisfiability, Chained Multicolored Clique and ACCEPTING NNCCM. These problems are called building blocks, as their main use is proving XNLP-hardness for many other problems (see Figure 1 .

Note that even though the XNLP-hardness of many problems in this paper are also (indirectly) reduced from List ColoRIng, this problem will be discussed in Section 4 as it is a well-studied problem with applications.

\subsection{Chained CNF-Satisfiability}

In this subsection we give a useful starting point for our transformations: a variation of SatisfiabiLity which we call Chained Weighted CNF-SATisfiability. The problem can be seen as a generalization of the $W$ [1]-hard problem WEIGHTED CNF-Satisfiability [15].

Chained Weighted CNF-Satisfiability

Input: $r$ disjoint sets of Boolean variables $X_{1}, X_{2}, \ldots X_{r}$, each of size $q$; integer $k \in \mathbf{N}$; Boolean formulas $F_{1}, F_{2}, \ldots, F_{r-1}$, where each $F_{i}$ is an expression in conjunctive normal form on variables $X_{i} \cup X_{i+1}$.

Parameter: $k$.

Question: Is it possible to satisfy the formula $F_{1} \wedge F_{2} \wedge \cdots \wedge F_{r}$ by setting exactly $k$ variables to true from each set $X_{i}$ and all others to false?

Our main result in this subsection is the following. We will also prove a number of variations later.

Theorem 3.1. Chained Weighted CNF-Satisfiability is XNLP-complete.

Proof. Membership in XNLP is easy to see. Indeed, for $i$ from 1 to $r$, we non-deterministically guess which variables in each $X_{i}$ are true, and keep the indexes of the true variables in memory for the two sets $X_{i}, X_{i+1}$. Verifying $F_{i}\left(X_{i}, X_{i+1}\right)$ can easily be done in logarithmic space and linear time. 
To show hardness, we transform from Timed Non-Deterministic CelluLar Automaton.

For each time step $t^{\prime}$, each cell $r \in[1, q]$, and each state $s \in S$, we have a Boolean variable $x_{t^{\prime}, r, s}$ with $x_{t^{\prime}, r, s}$ denoting whether the $r$ th cell of the automaton at time $t^{\prime}$ is in state $s$. For each time step $t^{\prime} \in[1, t-1]$, each cell $r \in[2, q]$, and each transition $z \in \mathcal{T}$ we have a variable $y_{t^{\prime}, r, z}$ that expresses that cell $r$ uses transition $z$ at time $t^{\prime}$.

We will build a Boolean expression and partitions of the variables, such that the expression is satisfiable by setting exactly $k$ variables to true from each set in the partition if and only if the machine can reach time step $t$ with at least one cell in accepting state starting from the initial configuration. The partition is based on the time of the automaton: for each time step $t^{\prime}$, the set $X_{t^{\prime}}$ consists of all variables of the form $x_{t^{\prime}, r, s}$ and $y_{t^{\prime}, r, s}$. For each set $X_{t^{\prime}}$ we require that exactly $2 q-2$ variables are set to true.

The formula has the following ingredients.

- At each step in time, each cell has exactly one state. Moreover, it uses a transition (unless at the first or final cell). To encode that we have at least one state, we use the expression $\bigvee_{s \in S} x_{t^{\prime}, r, s}$ for each $t^{\prime} \in[1, t-1]$ and $r \in[1, q]$. To encode that there is at least one transition, we use the clause $\bigvee_{z \in \mathcal{T}} y_{t^{\prime}, r, z}$ for all $t^{\prime} \in[1, t-1]$ and $r \in[2, q-1]$. Since we need to set exactly $2 q-2$ variables to true from $X_{t^{\prime}}$, and this is the total allowed amount, the pigeonhole principle shows that for each step in time $t^{\prime}$ and each cell $r$, at most (and hence, exactly) one state $s$ exists for which variable $x_{t^{\prime}, r, s}$ is true. Similarly, for all cells apart from the first and last, there is exactly one transition $z$ for which $y_{t^{\prime}, r, z}$ is true.

- We start in the initial configuration. We encode this using clauses with one literal $x_{0, r, s}$ whenever cell $r$ has state $s$ in the initial configuration.

- We end in an accepting state. This is encoded by

$$
\bigvee_{s \in A} \bigvee_{r} x_{t, r, s}
$$

- Left and right cells do not change. We add clauses $x_{t^{\prime}, 1, s_{L}}$ and $x_{t^{\prime}, q, s_{R}}$ with one literal for all time steps $t^{\prime}$.

- If a cell has a value at a time $t^{\prime}>0$, then there was a transition that caused it. This is encoded by

$$
x_{t^{\prime}, r, s} \Rightarrow \bigvee_{\left(s_{1}, s_{2}, s_{3}, s\right) \in \mathcal{T}} y_{t^{\prime}-1, r,\left(s_{1}, s_{2}, s_{3}, s\right)} .
$$

This is expressed in conjunctive normal form as

$$
\neg\left(x_{t^{\prime}, r, s}\right) \vee \bigvee_{\left(s_{1}, s_{2}, s_{3}, s\right) \in \mathcal{T}} y_{t^{\prime}-1, r,\left(s_{1}, s_{2}, s_{3}, s\right)} .
$$


- If a transition is followed, then the cell and its neighbors had the corresponding states. For each time step $t^{\prime} \in[1, t-1]$, cell $r \in[2, q-1]$ and transition $z=\left(s_{1}, s_{2}, s_{3}, s_{4}\right) \in \mathcal{T}$, we express this as

$$
y_{t^{\prime}, r, z} \Rightarrow\left(x_{t^{\prime}, r-1, s_{1}} \wedge x_{t^{\prime}, r, s_{2}} \wedge x_{t^{\prime}, r+1, s_{3}}\right) .
$$

We can rewrite this to the three clauses $\neg\left(y_{t^{\prime}, r, z}\right) \vee x_{t^{\prime}, r-1, s_{1}}$, and $\neg\left(y_{t^{\prime}, r, z}\right) \vee$ $x_{t^{\prime}, r, s_{2}}$, and $\neg\left(y_{t^{\prime}, r, z}\right) \vee x_{t^{\prime}, r+1, s_{3}}$. Since each 'inner' cell has $y_{t}$,

The last two steps ensure that the transition chosen from the $y$-variables agrees with the states chosen from $x$-variables.

It is not hard to see that we can build the formula with parameterized logarithmic space and in polynomial time, and that the formula is of the required shape.

A special case of the problem is when all literals that appear in the formulas $F_{i}$ are positive, i.e., we have no negations. We call this special case CHAINED Weighted Positive CNF-Satisfiability.

Theorem 3.2. Chained Weighted Positive CNF-Satisfiability is XNLPcomplete.

Proof. We modify the proof of the previous result. Note that we can replace each negative literal by the disjunction of all other literals from a set where exactly one is true, i.e., we may replace $\neg\left(x_{t^{\prime}, r, s}\right)$ and $\neg\left(y_{t^{\prime}, r, z}\right)$ by

$$
\bigvee_{s^{\prime} \neq s} x_{t^{\prime}, r, s^{\prime}} \text { and } \bigvee_{z^{\prime} \neq z} y_{t^{\prime}, r, z^{\prime}}
$$

respectively. The modification can be carried out in logarithmic space and polynomial time, and gives an equivalent formula. Thus, the result follows.

A closer look at the proof of Theorem 3.1 shows that $F_{2}=F_{3}=\cdots=$ $F_{r-2}$, and more specifically, we have a condition on $X_{1}$, a condition on $X_{r}$, and identical conditions on all pairs $X_{i} \cup X_{i+1}$ with $i$ from 1 to $r-1$. Thus, we also have XNLP-completeness of the following special case:

\section{Regular Chained Weighted CNF-Satisfiability}

Input: $r$ sets of Boolean variables $X_{1}, X_{2}, \ldots X_{r}$, each of size $q$; an integer $k \in \mathbf{N}$; Boolean formulas $F_{0}, F_{1}, F_{2}$ in conjunctive normal form, where $F_{0}$ and $F_{2}$ are expressions on $q$ variables, and $F_{1}$ is an expression on $2 q$ variables.

Parameter: $k$.

Question: Is it possible to satisfy the formula

$$
F_{0}\left(X_{1}\right) \wedge \bigwedge_{1 \leq i \leq r-1} F_{1}\left(X_{i}, X_{i+1}\right) \wedge F_{2}\left(X_{r}\right)
$$

by setting exactly $k$ variables to true from each set $X_{i}$ and all others to false? 
Also, the argument in the proof of Theorem 3.2 can be applied, and thus Regular Chained Weighted Positive CNF-Satisfiability (the variant of the problem above where all literals in $F_{0}, F_{1}$, and $F_{2}$ are positive) is XNLPcomplete.

For a further simplification of our later proofs, we obtain completeness for a regular variant without the first and last of the three formulas.

Regular Chained Weighted CNF-Satisfiability - II

Given: $r$ sets of Boolean variables $X_{1}, X_{2}, \ldots X_{r}$, each of size $q$; integer $k \in N$; Boolean formula $F_{1}$, which is in conjunctive normal form and an expression on $2 q$ variables.

Parameter: $k$.

Question: Is it possible to satisfy the formula

$$
\bigwedge_{1 \leq i \leq r-1} F_{1}\left(X_{i}, X_{i+1}\right)
$$

by setting exactly $k$ variables to true from each set $X_{i}$ and all others to false?

Theorem 3.3. Regular Chained Weighted CNF-Satisfiability - II is XNLP-complete.

Proof. The idea of the proof is to add the constraints from $F_{0}$ and $F_{2}$ to $F_{1}$, but to ensure that they are only 'verified' at the start and at the end of the chain.

To achieve this, we add variables $t_{i, j}$ for $i \in[1, r]$ and $j \in[1, r]$, with $t_{i, j}$ part of $X_{i}$. We increase the parameter $k$ by one. The construction is such that $t_{i, j}$ is true, if and only if $i=j ; t_{i, 1}$ implies all constraints from $F_{0}$, and $t_{i, r}$ implies all constraints from $F_{2}$. The details are as follows.

- We ensure that for all $i \in[1, r]$, exactly one $t_{i, j}$ is true. This can be done by adding a clause

$$
\bigvee_{1 \leq j \leq r} t_{i, j}
$$

As the number of disjoint sets of variables that each have at least one true variable still equals $k$ (as we increased both the number of these sets and $k$ by one), we cannot have more than one true variable in the set.

- For all $i \in[1, r]$ and $j \in[1, r]$, we enforce the constraint $t_{i, j} \Leftrightarrow t_{i+1, j+1}$ by adding the clauses $\neg t_{i, j} \vee t_{i+1, j+1}$ and $t_{i, j} \vee \neg t_{i+1, j+1}$.

- We add a constraint that ensures that $t_{i, 1}$ is false for all $i \in[2, r]$. This can be done by adding the clause with one literal $\neg t_{i+1,1}$ to the formula $F_{1}\left(X_{i}, X_{i+1}\right)$, i.e., we have a condition on a variable that is an element of the set given as second parameter. Together with the previous set of constraints, this ensures that $t_{i, 1}$ is true then $i=1$.

- Similarly, we add a constraint that ensures that $t_{i, r}$ is false for $i<r$. This is done by adding a clause with one literal $\neg t_{i, r}$ to $F_{1}\left(X_{i}, X_{i+1}\right)$. 
- We add a constraint of the form $t_{1} \rightarrow F_{0}(X)$ to $F_{1}$; for all $i$, the variable $t_{1}$ is substituted by $t_{i, 1}$ and $X$ by $X_{i}$.

- We add a constraint of the form $t_{r} \rightarrow F_{2}(X)$ to $F_{1}$; for all $i$, the variable $t_{1}$ is substituted by $t_{i, 1}$ and $X$ by $X_{i}$.

The first four additional constraints given above ensure that for all $i \in[1, r]$ and $j \in[1, r], t_{i, j}$ is true if and only if $i=j$. Thus, the fifth constraint enforces $F_{0}\left(X_{1}\right)$ (since $t_{1,1}$ has to be true); for $i>1$, this constraint has no effect. Likewise, the sixth constraint enforces $F_{2}\left(X_{r}\right)$. Hence, the new set of constraints is equivalent to the constraints for the first version of REGULAR Chained Weighted CNF-Satisfiability.

Using standard logic operations, the constraints can be transformed to conjunctive normal form; one easily can verify the time and space bounds.

Again, with a proof identical to that of Theorem 3.2, we can show that the variant with only positive literals (Regular Chained Weighted Positive CNF-SatisfiabiLITY - II) is XNLP-complete.

From the proofs above, we note that each set of variables $X_{i}$ can be partitioned into $k$ subsets, and a solution has exactly one true variable for each subset, e.g., for each $t^{\prime}$, exactly one $x_{t^{\prime}, r, s}$ is true in the proof of Theorem 3.1 This still holds after the modification in the proofs of the later results. We define the following variant.

Partitioned Regular Chained Weighted CNF-Satisfiability

Input: $r$ sets of Boolean variables $X_{1}, X_{2}, \ldots X_{r}$, each of size $q$; an integer $k \in N$; Boolean formula $F_{1}$, which is in conjunctive normal form and an expression on $2 q$ variables; for each $i$, a partition of $X_{i}$ into $X_{i, 1}, \ldots, X_{i, k}$ with for all $i_{1}, i_{2}, j:\left|X_{i_{1}, j}\right|=$ $\left|X_{i_{2}, j}\right|$.

Parameter: $k$.

Question: Is it possible to satisfy the formula

$$
\bigwedge_{1 \leq i \leq r-1} F_{1}\left(X_{i}, X_{i+1}\right)
$$

by setting from each set $X_{i, j}$ exactly 1 variable to true and all others to false?

We call the variant with only positive literals PARTitioned Regular Chained Weighted Positive CNF-SAtisfiability.

Corollary 3.4. Partitioned Regular Weighted Chained CNF-Satisfiability and Partitioned Regular Chained Weighted Positive CNF-Satisfiability are XNLP-complete. 


\subsection{Chained Multicolored Clique}

The Multicolored Clique problem is an important tool to prove fixed parameter intractability of various parameterized problems. It was independently introduced by Pietrzak [38] (under the name Partitioned Clique) and by Fellows et al. [23.

In this paper, we introduce a chained variant of Multicolored Clique. In this variant, we ask to find a sequence of cliques, that are overlapping with the previous and next clique in the chain.

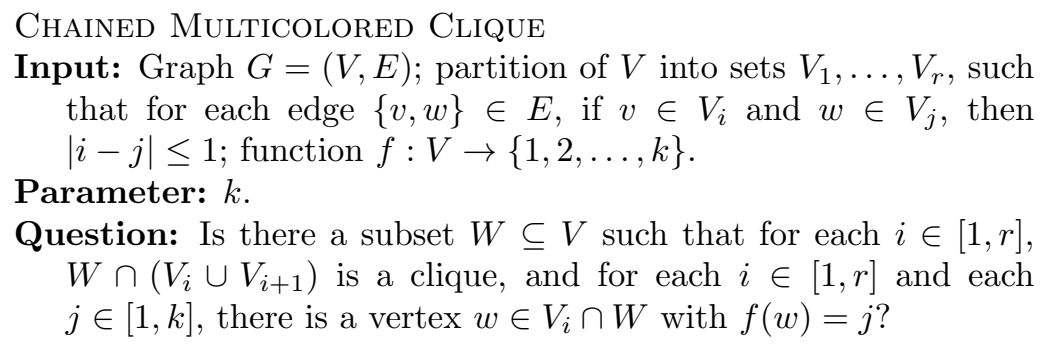

Thus, we have a clique with $2 k$ vertices in $V_{i} \cup V_{i+1}$ for each $i \in[1, r-1]$, with for each color a vertex with that color in $V_{i}$ and a vertex with that color in $V_{i+1}$. Importantly, the same vertices in $V_{i}$ are chosen in the clique for $V_{i-1} \cup V_{i}$ as for $V_{i} \cup V_{i+1}$ for each $i \in[2, r-1]$. Below, we call such a set a chained multicolored clique.

\section{Theorem 3.5. Chained Multicolored Clique is XNLP-complete.}

Proof. Membership in XNLP is easy to see: iteratively guess for each $V_{i}$ which vertices belong to the clique. We only need to keep the clique vertices in $V_{i-1}$ and $V_{i}$ in memory.

We now prove hardness via a transformation from LiST COLORING parameterized by pathwidth (which is proved XNLP-complete in Subsection 4.1). Suppose that we are given a graph $G=(V, E)$, a path decomposition $\left(X_{1}, \ldots, X_{r}\right)$ of width $k$ for $G$ and a list of colors $L_{v}$ for each vertex $v \in V$.

First, we build an equivalent instance of LIST COLORING parameterized by pathwidth with the property that all the sets $X_{i}$ have size exactly $k+1$. This can be done by adding $k+1-\left|X_{i}\right|$ new isolated vertices to each set $X_{i}$ with $\left|X_{i}\right|<k+1$, which obtain the color list $\{1\}$.

From this, we build an instance of Chained Multicolored Clique as follows. For each $X_{i}$, we choose a bijection $f_{i}: X_{i} \rightarrow\{1, \ldots, k+1\}$. For each vertex $v \in X_{i}$ and each color $c \in S_{v}$, we create a vertex $w_{i, v, c}$ in $V_{i}$ and color it $f\left(w_{i, v, c}\right)=f_{i}(v)$. Thus, for each vertex $v$, we have created a vertex for each combination $(c, i)$ of a possible color and possible bag $X_{i}$ for the vertex to appear. We have the following edges.

- We have an edge $\left\{w_{i, v, c}, w_{i, v^{\prime}, c^{\prime}}\right\}$ if $c \neq c^{\prime}$ or $\left\{v, v^{\prime}\right\} \notin E$, for all $v, v^{\prime} \in X_{i}$ with $v \neq v^{\prime}, c \in S_{v}$, and $c^{\prime} \in S_{v^{\prime}}$. 
- We have an edge $\left\{w_{i, v, c}, w_{i+1, v, c}\right\}$ for all $v \in X_{i} \cap X_{i+1}$.

- We have an edge $\left\{w_{i, v, c}, w_{i+1, v^{\prime}, c^{\prime}}\right\}$ if $c \neq c^{\prime}$ or $\left\{v, v^{\prime}\right\} \notin E$, for all $v \in X_{i}$, $v^{\prime} \in X_{i+1}$ with $v \neq v^{\prime}, c \in S_{v}$, and $c^{\prime} \in S_{v^{\prime}}$.

Let $H=\left(V_{1}, \ldots, V_{r}\right)$ be the resulting graph (with coloring $f$ ).

Claim 3.6. $H$ has a chained multicolored clique if and only if $G$ has a list coloring.

Proof. Suppose that $G$ has a list coloring $c$. For each $i \in[1, r]$ and $v \in X_{i}$, select the vertex $w_{i, v, c(v)}$. It is easy to verify that the resulting set is a chained multicolored clique.

Suppose now that $H$ has a chained multicolored clique $S$. First, note that for each $i \in[1, r]$ and $v \in X_{i}$, there is exactly one vertex of the form $w_{i, v, \gamma}$ in $S$. There can be at most one such vertex because $\left\{w_{i, v, \gamma}, w_{i, v, \gamma^{\prime}}\right\}$ is not an edge of $H$, and there has to be at least one since all vertices of color $f_{i}(v)$ in $V_{i}$ are of the form $w_{i, v, \gamma}$.

Consider a vertex $v \in X_{i} \cap X_{i+1}$. Let $\gamma, \gamma^{\prime}$ be such that $w_{i, v, \gamma}, w_{i+1, v, \gamma^{\prime}} \in S$. If $\gamma \neq \gamma^{\prime}$, then $w_{i, v, \gamma}$ is not adjacent to $w_{i+1, v, c \gamma^{\prime}}$. So we can define $c(v)$ as the unique $\gamma$ for which $w_{i, v, \gamma} \in S$ for $i$ such that $v \in X_{i}$. Note that $c(v)$ is in the list of $v$.

It remains to show that the function $c$ is a proper coloring. Consider an edge $\left\{v, v^{\prime}\right\} \in E$. By the definition of path decomposition, there is a bag $X_{i}$ with $v, v^{\prime} \in X_{i}$. By the discussion above, both $w_{i, v, c(v)} \in S$ and $w_{i, v^{\prime}, c\left(v^{\prime}\right)} \in S$, and so these vertices are adjacent. As $\left\{v, v^{\prime}\right\} \in E$, the construction of $H$ shows that we must have $c(v) \neq c\left(v^{\prime}\right)$. This implies that $c$ is a proper coloring.

It is not hard to see that $H$ and $f$ can be constructed in logarithmic space and fpt time; the result follows.

A simple variation is the following. We are given a graph $G=(V, E)$, a partition of $V$ into sets $V_{1}, \ldots, V_{r}$ with the property that for each edge $\{v, w\} \in E$, if $v \in V_{i}$ and $w \in V_{j}$ then $|i-j| \leq 1$, and a coloring function $f: V \rightarrow\{1,2, \ldots, k\}$. A chained multicolored independent set is an independent set $S$ with the property that for each $i \in[1, r]$ and each color $j \in[1, k]$, the set $S$ contains exactly one vertex $v \in V_{i}$ of color $f(v)=j$. The Chained Multicolored Independent SET problem asks for the existence of such a chained multicolored independent set, with the number of colors $k$ as parameter. We have the following simple corollary.

Corollary 3.7. Chained Multicolored Independent Set is XNLP-complete.

Proof. This follows directly from Theorem 3.5, by observing that the following 'partial complement' of a partitioned graph $G=\left(V_{1} \cup \cdots V_{r}, E\right)$ can be constructed in logarithmic space: create an edge $\{v, w\}$ if and only if there is an $i$ with $v \in V_{i}$ and $w \in V_{i} \cup V_{i+1}, v \neq w$ and $\{v, w\} \notin E$. 


\subsection{Non-decreasing counter machines}

In this subsection, we introduce a new simple machine model, which can also capture the computational power of XNLP (see Theorem 3.8). This model will be a useful stepping stone when proving XNLP-hardness reductions in Section 4

A Nondeterministic Nonincreasing Checking Counter Machine (or: NNCCM) is described by a 3-tuple $(k, n, s)$, with $k$ and $n$ positive integers, and $s=$ $\left(s_{1}, \ldots, s_{r}\right)$ a sequence of 4 -tuples (called checks). For each $i \in\{1, \ldots, r\}$, the 4 -tuple $s_{i}$ is of the form $\left(c_{1}, c_{2}, r_{1}, r_{2}\right)$ with $c_{1}, c_{2} \in\{1,2, \ldots, k\}$ positive integers and $r_{1}, r_{2} \in\{0,1,2, \ldots, n\}$ non-negative integers. These model the indices of the counters and their values respectively.

An NNCCM $(k, n, s)$ with $s=\left(s_{1}, \ldots, s_{r}\right)$ works as follows. The machine has $k$ counters that are initially 0 . For $i$ from 1 to $r$, the machine first sets each of the counters to any integer that is at least its current value and at most $n$. After this, the machine performs the $i$ th check $s_{i}=\left(c_{1}, c_{2}, r_{1}, r_{2}\right)$ : if the value of the $c_{1}$ th counter equals $r_{1}$ and the value of the $c_{2}$ th counter equals $r_{2}$, then we say the $i$ th check rejects and the machine halts and rejects. When the machine has not rejected after all $r$ checks, the machine accepts.

The nondeterministic steps can be also described as follows. Denote the value of the $c$ th counter when the $i$ th check is done by $c(i)$. We define $c(0)=0$. For each $i \in\{1, \ldots, r\}, c(i)$ is an integer that is nondeterministically chosen from $[c(i-1), n]$.

We consider the following computational problem.

ACCEPTING NNCCM

Given: An NNCCM $(k, n, s)$ with all integers given in unary.

Parameter: The number of counters $k$.

Question: Does the machine accept?

Theorem 3.8. ACCEPTING NNCCM is XNLP-complete.

Proof. We first argue that ACCEPTING NNCCM belongs to XNLP. We simulate the execution of the machine. At any point, we store $k$ integers from $[0, n]$ that give the current values of our counters, as well as the index $i \in[1, r]$ of the check that we are performing. This takes only $O(k \log n+\log r)$ bits. When we perform the check $s_{i}=\left(c_{1}, c_{2}, r_{1}, r_{2}\right)$, we store the values $c_{1}, c_{2}, r_{1}, r_{2}$ in order to perform the check using a further $O(\log n)$ bits. So we can simulate the machine using $O(k \log n+\log r)$ space. The running time is upper bounded by some function of the form $f(k) \cdot \operatorname{poly}(n, r)$.

We now prove hardness via a transformation from CHAined Multicolored Clique. We are given a $k$-colored graph with vertex sets $V_{1}, \ldots, V_{r}$. By adding isolated vertices if needed, we may assume that, for each $i \in[1, r]$, the set $V_{i}$ contains exactly $m$ vertices of each color. We will assume that $r$ is even; the proof is very similar for odd $r$. We set $n=m r$.

We create $4 k$ counters: for each colour $i \in[1, k]$, there are counters $c_{i, 1,+}$, $c_{i, 1,-}, c_{i, 0,+}, c_{i, 0,-}$. We use the counters $c_{i, 1, \pm}$ for selecting vertices from sets $V_{j}$ with $j$ odd and the counters $c_{i, 0, \pm}$ for selecting vertices from sets $V_{j}$ with $j$ even. 
The intuition is the following. We increase the counters in stages, where in stage $j$ we model the selection of the vertices from $V_{j}$. Say $j$ is even. We increase the counters $c_{i, 0,+}, c_{i, 0,-}$ to values within $[j m+1,(j+1) m]$. Since counters may only move up, there can be at most one $\ell \in[1, m]$ for which the counters at some point take the values $c_{i, 0,+}=j m+\ell$ and $c_{i, 0,-}=(j+1) m+1-\ell$. We enforce that such an $\ell$ exists and interpret this as placing the $\ell$ th vertex of color $i$ in $V_{j}$ into the chained multicolored clique.

We use the short-cut $\left(c_{1}, c_{2}, R_{1}, R_{2}\right)$ for the sequence of checks $\left(\left(c_{1}, c_{2}, r_{1}, r_{2}\right)\right.$ : $r_{1} \in R_{1}, r_{2} \in R_{2}$ ) performed in lexicographical order, e.g. for $R_{1}=\{1,2\}$ and $R_{2}=\{1,2,3\}$, the order is $(1,1),(1,2),(1,3),(2,1),(2,2),(2,3)$.

For each $j \in[1, r]$, the $j$ th vertex selection check confirms that for each $i \in[1, k],\left(c_{i, p a r,+}, c_{i, p a r,-}\right)$ is of the form $(j m+\ell,(j+1) m+1-\ell)$ for some $\ell \in$ $[1, m]$, where par denotes the parity of $j$. For each $i \in[1, k]$, we set $c_{1}=c_{i, p a r,+}$ and $c_{2}=c_{i, p a r,-}$, and perform the following checks in order.

1. $\left(c_{1}, c_{2},[0, j m-1],[0, n]\right)$;

2. $\left(c_{1}, c_{2},[0, n],[0, j m-1]\right)$;

3. for $\ell \in[1, m],\left(c_{1}, c_{2},\{j m+\ell\},[j m,(j+1) m] \backslash\{(j+1) m+1-\ell\}\right)$;

4. $\left(c_{1}, c_{2},[(j+1) m+1, n],[0, n]\right)$;

5. $\left(c_{1}, c_{2},[0, n],[(j+1) m+1, n]\right)$.

Suppose all the checks succeed. After the second check, $c_{1}$ and $c_{2}$ are both at least $j m$, and before the last two checks, $c_{1}$ and $c_{2}$ are both at most $(j+1) m$. The middle set of checks ensure that there is some $\ell$ for which the $c_{1}$ th counter and $c_{2}$ th counter have been simultaneously at the values $j m+\ell$ and $(j+1) m+1-\ell$ respectively. We say the check chooses $\ell$ for $c_{1}$ and $c_{2}$. Since the counters can only move up, this $\ell$ is unique.

This is used as a subroutine below, where we create a collection of checks such that the corresponding NNCCM accepts if and only if the $k$-colored graph has a chained multicolored clique. For $j=1$ to $r$, we do the following.

- Let par $\equiv j \bmod 2$ denote the parity of $j$ and let par' $\in\{0,1\}$ denote the opposite parity.

- We perform a $j$ th vertex selection check.

- We verify that all selected vertices in $V_{j}$ are adjacent. Let $u u^{\prime}$ be a nonedge with $u, u^{\prime} \in V_{j}$. Let $\ell, i, \ell^{\prime}, i^{\prime}$ with $i \neq i^{\prime}$ be such that $u$ is the $\ell$ th vertex of color $i$ in $V_{j}$ and $u^{\prime}$ is the $\ell^{\prime}$ th vertex of color $i^{\prime}$. We add the check $\left(c_{i, p a r,+}, c_{i^{\prime}, \text { par },+}, j m+\ell, j m+\ell^{\prime}\right)$. This ensures that we do not put both $u$ and $u^{\prime}$ in the clique of $V_{j}$.

- If $j>1$, then we verify that all selected vertices in $V_{j}$ are adjacent to all selected vertices in $V_{j-1}$. Let $u u^{\prime}$ be a non-edge with $u \in V_{j}$ and $u^{\prime} \in V_{j-1}$ and let $\ell, i, \ell^{\prime}, i^{\prime}$ be as above. We add the check $\left(c_{i, p a r,+}, c_{i^{\prime}, p a r^{\prime},+}, j m+\right.$ $\left.\ell, j m+\ell^{\prime}\right)$ to ensure that we do not put both $u$ and $u^{\prime}$ into the clique. 
- We finish with another $j$ th vertex selection check. If $j>1$, we also do a $(j-1)$ th vertex selection check. This ensures our counters are still 'selecting vertices' from $V_{j-1}$ and $V_{j}$.

We now argue that the set of checks created above accepts if and only if the graph contains a chained multicolored clique. Suppose first that such a set $W \subseteq V$ exists for which $W \cap\left(V_{j} \cup V_{j+1}\right)$ forms a clique for all $j$ and $W$ contains at least one vertex from $V_{j}$ of each color. We may assume that $W_{j}=W \cap V_{j}$ is of size $k$ for each $j$. Let $j \in[1, r]$ be given of parity par and for each $i \in[k]$, let the $f(i)$ th vertex of $V_{j}$ of color $i$ be in $W_{j}$. Before the first $j$ th vertex selection check, we move the counters $\left(c_{i, p a r,+}, c_{i, p a r,-}\right)$ to $(j m+f(i),(j+1) m-1-f(i))$, and these will be left there until the first $(j+2)$ th vertex selection check. This will ensure that all the checks accept.

Suppose now that all the checks accept. We first make an important observation. Let two counters $c_{1}$ and $c_{2}$ be given. If in an iteration above, the first vertex selection check chooses $\ell$ for $c_{1}$ and $c_{2}$ and the second vertex selection check chooses $\ell^{\prime}$, then it must be the case that $\ell=\ell^{\prime}$. Indeed, we cannot increase $c_{1}$ or $c_{2}$ beyond $(j+1) m$ before the last vertex selection check, and they need to be above $j m$ due to the first. If the first vertex selection check selects $\ell$, then the $c_{1}$ the counter is at least $j m+\ell$, so in order for it to be $j m+\ell^{\prime}$ in the second check, we must have $\ell^{\prime} \geq \ell$. Considering the value of the $c_{2}$ th counter, we also find $\ell \geq \ell^{\prime}$ and hence $\ell=\ell^{\prime}$. In particular, the counters cannot have moved between the two vertex selection checks.

It is hence well-defined to, for $j \in[1, r]$ of parity par, let $W_{j}$ be the set of vertices that are for some color $i \in[1, k]$ the $\ell$ th vertex of color $i$ in $V_{j}$, for $\ell$ the unique value that is selected by a $j$ th vertex selection check for $c_{i, p a r,+}$ and $c_{i, p a r,-}$. We claim that $W=\cup_{j=1}^{r} W_{j}$ is our desired multicolored chained clique. It contains exactly one vertex per color from $V_{j}$. Suppose $u \in W_{j}$ and $u^{\prime} \in W_{j-1} \cup W_{j}$ are not adjacent and distinct. Let $i$,par, $\ell$ be such that $u$ is the $\ell$ th color of parity par in $V_{j}$ for $j$ of parity par, and similar for $i^{\prime}$, par $^{\prime}, \ell^{\prime}$. Then at the $j$ th iteration, the check $\left(c_{i, p a r,+}, c_{i^{\prime}, p a r,+}, j m+\ell, j m+\ell^{\prime}\right)$ has been performed (because $u u^{\prime}$ is a non-edge). Since $u, u^{\prime} \in W$ and this check is done between vertex selection checks, the counters have to be at those values. This shows that there is a check that rejects, a contradiction. So $W$ must be a chained multicolored clique.

The ACCEPTING NNCCM problem appears to be a very useful tool for giving XNLP-hardness proofs. Note that one step where the $k$ counters can be increased to values at most $n$ can be replaced by $k n$ steps where counters can be increased by one, or possibly a larger number, again to at most $n$. This modification is used in some of the proofs in the following section.

\section{Applications}

In this section, we consider several problem, which we prove to be XNLPcomplete. 
We start by proving XNLP-completeness for LIST COLORING parameterized by pathwidth in Subsection 4.1 and Dominating Set parameterized by logarithmic pathwidth in Subsection 4.2. We continue to prove XNLP-completeness for a well-studied scheduling problem, Scheduling with Precedence ConSTRAINTS parameterized by the number of machines and the partial order width in Subsection 4.3. Next, in Subsection 4.4 we discuss Uniform Emulations of Weighted PAths and use it to prove XNLP-completeness for BANDWIDTH in Subsection 4.5. We end the section with proving Timed Dominating Set RECONFIgUration to be XNLP-complete in Subsection 4.6 .

Note that some variants of these problems are discussed in Appendix Band Appendix C.

\subsection{List Coloring Parameterized by Pathwidth}

In this subsection, we consider the List Coloring and Precoloring ExTENSION problems, parameterized by treewidth or pathwidth. These problems belong to XP 32. Fellows et al. 22, have shown that List Coloring and PreCOlORING EXTEnsion parameterized by treewidth are $W[1]$-hard. (The transformation in their paper also works for parameterization by pathwidth.) We strengthen this result by showing that List COLORING and PRECOLORING ExTENSION parameterized by pathwidth are XNLP-complete. Note that this result also implies $W[t]$-hardness of the problems for all integers $t$.

Given a graph $G=(V, E)$ with lists $L_{v}$ for vertex $v \in V(G)$, a list coloring for $G$ is a choice of color $f(v) \in L_{v}$ for each vertex $v$ such that $f(v) \neq f(w)$ when $\{v, w\} \in E$.

A path decomposition of a graph $G=(V, E)$ is a sequence $\left(X_{1}, X_{2}, \ldots, X_{r}\right)$ of subsets of $V$ with the following properties.

1. $\bigcup_{1 \leq i \leq r} X_{i}=V$.

2. For all $\{v, w\} \in E$, there is an $i \in I$ with $v, w \in X_{i}$.

3. For all $1 \leq i_{0}<i_{1}<i_{2} \leq r, X_{i_{0}} \cap X_{i_{2}} \subseteq X_{i_{1}}$.

The width of a path decomposition $\left(X_{1}, X_{2}, \ldots, X_{r}\right)$ equals $\max _{1 \leq i \leq r}\left|X_{i}\right|-1$, and the pathwidth of a graph $G$ is the minimum width of a path decomposition of $G$.

In this subsection, we assume that a path decomposition of width at most $k$ is given as part of the input.

Theorem 4.1. LIST COLORING parameterized by pathwidth is XNLP-complete.

Proof. The proof that the problem is in XP for bounded treewidth by Jansen and Scheffler 32] can easily be transferred to a proof that the problem is in XNLP. Intuitively, we guess which of the table entries in the DP table for each bag in the path decomposition we take; we store one table entry in $O(\operatorname{pw} \log n)$ 
bits. (Each table entry for a bag gives the choice of colors for the vertices in that bag.)

To show hardness, we transform from Monotone Partitioned Chained CNF-SATisfiability.

Suppose we have an instance of Monotone Partitioned Chained CNFSATisfiability, say, variable sets $X_{i, j}$ with $i \in[1, r]$ and $j \in[1, k]$, each of size $q$ and a formula $F$ (on $2 k q$ Boolean variables), and we ask if we can set exactly one variable from each set $X_{i, j}$ to true, such that

$$
\bigvee_{i=1}^{r-1} F\left(X_{i, 1}, \ldots X_{i, k}, X_{i+1,1}, \ldots, X_{i+1, k}\right)
$$

holds, with $F$ in conjunctive normal form with only positive literals.

We build a graph $G$, a path decomposition of width $2 k+1$ of $G$, and an assignment of colors to vertices of $G$ as follows.

First, for every set $X_{i, j}$ we create a vertex $v_{i, j}$, and for every variable in $X_{i, j}$ we create a color in the list of colors for the vertex $v_{i, j}$. The $i$ th bag in the path decomposition consists of all the vertices $v_{i, j}$ and $v_{i+1, j}$ (for all $j$ ). Some additional vertices and bags will now be added in order to model the clauses.

Fix $i \in[1, r-1]$. Consider a clause $c$, which is a conjunction of positive literals, on the set of variables $S_{i}=\bigcup_{j} X_{i, j} \cup X_{i+1, j}$. Let $x \in X_{i, j}$ be such that $x$ does not appear in $c$. We create a new vertex $w_{c, x}$ adjacent to $v_{i, j}$ with color list $\left\{x, n_{i, j}\right\}$; the colors $n_{i, j}$ are new. We create one further new vertex $z_{c}$ for the clause $c$ that is adjacent to all vertices $w_{c, x}$ and whose color list contains the colors $n_{i, j}$ and $n_{i+1, j}$ for all $j$.

Claim 4.2. There is a satisfying truth assignment fulfilling the conditions of the Monotone Partitioned Chained CNF-SATisfiability problem, if and only if there is a proper coloring where each vertex has a color from its list.

Proof. Suppose that a satisfying truth assignment exists. Color a vertex $v_{i, j}$ with the color of the variable from the set $X_{i, j}$ which is set to true. Consider a vertex $w_{c, x}$ with $c$ a clause and $x \in X_{i, j}$ a literal that does not appear in the clause $c$. We color $w_{c, x}$ with the color $x$ if $x$ is set to false, and with the color $n_{i, j}$ if $x$ is set to true. This avoids conflicts with the color of its neighbor $v_{i, j}$.

For each vertex $z_{c}$, there must be a literal that satisfies $c$, say $x \in X_{i, j}$. This implies that all $x^{\prime} \in X_{i, j} \backslash\{x\}$ are set to false, and thus no neighbor of $z_{c}$ is colored with $n_{i, j}$. Thus, we can color $z_{c}$ with $n_{i, j}$.

Conversely, suppose that a list coloring of $G$ exists. We define a truth assignment by setting a variable $x \in X_{i, j}$ to true, if and only if the color of $v_{i, j}$ is $x$. This selects one true variable per set $X_{i, j}$. It remains to verify that each clause is satisfied.

Consider a clause $c$. Let $n_{i, j}$ be the color of $z_{c}$ and let $x \in X_{i, j}$ be the color of $v_{i, j}$. It must be that $x \in c$ : if $x$ does not appear in $c$, then the vertex $w_{c, x}$ exists and cannot be colored, since the color $x$ has already been used for its neighbor $v_{i, j}$ and the color $n_{i, j}$ has already been used for its neighbor $z_{c}$. As $x \in c$ and 
the color of $v_{i, j}$ is $x$, we find that $c$ is satisfied by the truth assignment. This shows that each clause is satisfied, and finishes the proof of the claim.

We now discuss how to build a path decomposition of width $2 k+1$ for $G$. The procedure has three nested loops - for each, we can use $O(\log n)$ bits to denote where in the loop we are. The resulting path decomposition is the concatenation of the bags, generated in the order implied by the loops.

The outermost loop goes through the 'time steps': we loop $i$ from 1 to $r-1$. The middle loop goes through the clauses for step $i$, i.e., all clauses $c$ with variable set $\bigcup_{j}\left(X_{i, j} \cup X_{i+1, j}\right)$. The innermost loop deals with one clause $c$ with variables from the set $\bigcup_{j}\left(X_{i, j} \cup X_{i+1, j}\right)$. For each variable $x \in \bigcup_{j}\left(X_{i, j} \cup X_{i+1, j}\right)$ that does not appear in $c$, we take a bag with vertex set

$$
\left\{z_{c}, w_{c, x}\right\} \cup \bigcup_{j=1}^{k}\left\{v_{i, j}, v_{i+1, j}\right\} .
$$

Each bag has size at most $2 k+2$; moreover, it is easy to see that the procedure gives a path decomposition.

As we can construct the graph $G$, the color lists and the path decomposition using $O(k \log n)$ bits in memory, the result now follows.

We deduce the following result from a well-known transformation from LisT COLORING.

Corollary 4.3. PRE-COLORING EXTENSION parameterized by pathwidth is XNLPcomplete.

Proof. Consider an instance of List Coloring with graph $G=(V, E)$ and color lists $L_{v}$ for all $v \in V$. Let $\mathcal{C}=\bigcup_{v \in V} L_{v}$ be the set of all colors. For each vertex $v$ with color list $L_{v}$, for each color $\gamma \in \mathcal{C} \backslash L_{v}$, add a new vertex that is only adjacent to $v$ and is precolored with $\gamma$. The original vertices are not precolored. It is easy to see that we can extend the precoloring of the resulting graph to a proper coloring, if and only if the instance of LiST COLORING has a solution.

The procedure increases the pathwidth by at most one. Indeed, if we are given a path decomposition of $G$, then we can build the path decomposition of the resulting graph as follows. We iteratively visit all the bags $X_{i}$ of the path decomposition. Let $v \in X_{i}$ be a vertex for which $X_{i}$ is the first bag it appears in (so $i=1$ or $v \notin X_{i-1}$ ). We create a new bag $X_{i} \cup\{w\}$ for each new neighbor $w$ of $v$ and place this bag somewhere in between $X_{i}$ and $X_{i+1}$. This transformation can be easily executed with $O(k \log n)$ bits of memory.

Note that we assume that a path decomposition of the input graph $G$ is given as part of the input. We conjecture that such a path decomposition can be found with a non-deterministic algorithm using logarithmic space and 'fpt' time, but the details need a careful study. Elberfeld et al. 20] show that for each fixed $k$, determining if the treewidth is at most $k$, and if so, finding a tree decomposition of width at most $k$ belongs to $L$. 
From Theorem 4.1 and Corollary 4.3 , we can also directly conclude that List Coloring and PRECOLORING EXTENSION are XNLP-hard when parameterized by the treewidth. However, we leave membership of these problems when parameterized by treewidth as an open problem.

\subsection{Logarithmic Pathwidth}

There are several well known problems that can be solved in time $O\left(c^{k} n\right)$ for a constant $c$ on graphs of pathwidth or treewidth at most $k$. Classic examples are IndePendent Set and Dominating Set (see e.g., [14, Chapter 7.3]), but there are many others, e.g., [5, 44.

In this subsection, rather than bounding the pathwidth by a constant, we allow the pathwidth to be linear in the logarithm of the number of vertices of the graph. We consider the following problem.

Log-Pathwidth Dominating Set

Input: Graph $G=(V, E)$, path decomposition of $G$ of width $\ell$, integer $K$.

Parameter: $\lceil\ell / \log |V|\rceil$.

Question: Does $G$ have a dominating set of size at most $K$ ?

In Appendix C, we study the independent set and clique variants of this problem.

Theorem 4.4. Log-Pathwidth Dominating Set is XNLP-complete.

Proof. It is easy to see that the problem is in XNLP - run the standard dynamic programming algorithm for Dominating SET on graphs with bounded pathwidth, but instead of computing full tables, guess the table entry for each bag.

Hardness for XNLP is shown with help of a reduction from PARTITIONED Regular Chained Weighted CNF-Satisfiability.

Suppose we are given $F_{1}, X_{1}, \ldots, X_{r}, q, k, X_{i, j}(1 \leq i \leq r, 1 \leq j \leq$ $k)$ as instance of the Partitioned Regular Chained Weighted CNFSATISFiability problem.

We assume that each set $X_{i, j}$ is of size $2^{t}$ for some integer $t$; otherwise, add dummy variables to the sets and a clause for each $X_{i, j}$ that expresses that a non-dummy variable from the set is true. Let $c$ be the number of clauses in $F_{1}$. We now describe in a number of steps the construction of $G$.

Variable choice gadget For each set $X_{i, j}$ we have a variable choice gadget. The gadget consists of $t$ copies of a $K_{3}$. Each of these $K_{3}$ 's has one vertex marked with 0 , one vertex marked with 1 , and one vertex of degree two, i.e., this latter vertex has no other neighbors in the graph.

The intuition is the following. We represent each element in $X_{i, j}$ by a unique $t$-bit bitstring (recall that we ensured that $\left|X_{i, j}\right|=2^{t}$ ). Each $K_{3}$ represents one bit, and together these bits describe one element of $X_{i, j}$, namely the variable that we set to be true. 


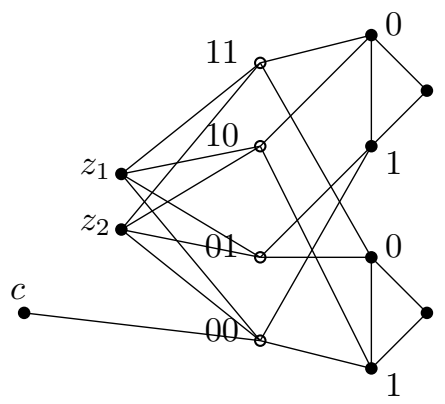

Figure 2: Example of clause gadget. Here, $t=2$ and only the variable with index 00 satisfies clause $c$

Clause checking gadget Consider a clause $\phi$ on $X_{i} \cup X_{i+1}$. We perform following construction separately for each such clause, and in particular the additional vertices defined below are not shared among clauses. For each $j \in$ $[1, k]$, we add $2^{t}+2$ additional vertices for both $X_{i, j}$ and $X_{i+1, j}$, as follows.

- We create a variable representing vertex $v_{x}$ for each variable $x \in X_{i, j} \cup$ $X_{i+1, j}$. We connect $v_{x}$ to the each vertex that represents a bit in the complement of the bit string representation of $x$. We call this vertex a .

- We add two new vertices for $X_{i, j}$ and also for $X_{i+1, j}$ (see $z_{1}$ and $z_{2}$ in Figure 2). These vertices are incident to all variable representing vertices for variables from the corresponding set.

We call this construction a variable set gadget.

The clause checking gadget for $\phi$ consists of $2 k$ variable set gadgets (for each $j \in[1, k]$, one for each set of the form $X_{i, j}$ and $X_{i+1, j}$ ), and one additional clause vertex. The clause vertex is incident to all vertices in the current gadget that represent a variable that satisfies that clause. In total, we add $2 \cdot k \cdot\left(2^{t}+2\right)+1$ variables per clause.

The intuition is the following. From each triangle, we place either the vertex marked with 0 or with 1 in the dominating set. This encodes a variable for each $X_{i, j}$ as follows. The vertices in the triangles dominate all but one of the variable representing vertices; the one that is not dominated is precisely the encoded variable. This vertex is also placed in the dominating set (we need to pick at least one variable representing vertex in order to dominate the vertices $z_{1}$ and $z_{2}$ private to $\left.X_{i, j}\right)$ ). The clause is satisfied exactly when the clause vertex has a neighbor in the dominating set.

The graph $G$ is formed by all variable choice and clause checking gadgets, for all variables and clauses. Recall that $c$ is the number of clauses in $F_{1}$.

Claim 4.5. There is a dominating set in $G$ of size $r k t+2 k c(r-1)$, if and only if the instance of PARtitioned Regular Chained Weighted CNFSATISFIABILITY has a solution. 
Proof. Suppose we have a solution for the latter. Select in the variable choice gadgets the bits that encode the true variables and put these in the dominating set $S$. As we have $r k$ sets of the form $X_{i, j}$, and each is represented by $t$ triangles of which we place one vertex per triangle in the dominating set, we already used rkt vertices.

For each clause, we have $2 k$ sets of the form $X_{i, j}$. For each, take the vertex that represents the true variable of the set and place it in $S$. Note that all vertices that represent other variables are dominated by vertices from the variable choice gadget. For each set $X_{i, j}$, the chosen variable representing vertex dominates the vertices marked $z_{1}$ and $z_{2}$. As the clause is satisfied, the clause vertex is dominated by the vertex that represents the variable representing vertex for the variable that satisfies it. This shows $S$ is a dominating set. We have $(r-1) c$ clauses in total, and for each we have $2 k$ sets of the form $X_{i, j}$. We placed one variable representing vertex for each of these $(r-1) c \cdot 2 k$ sets in the dominating set. In total, $|S|=r k t+2 k c(r-1)$ as desired.

For the other implication, suppose there is a dominating set $S$ of size $r k t+$ $2 k c(r-1)$.

We must contain one vertex from each triangle in a variable choice gadget (as the vertex with degree two must be dominated). Thus, these gadgets contain at least $r k t$ vertices from the dominating set.

Each of the vertices marked $z_{1}$ and $z_{2}$ must be dominated. So we we must choose at least one variable choice vertex per variable set gadget. This contributes at least $2 k c(r-1)$ vertices.

It follows that we must place exactly those vertices and no more: the set $S$ contains exactly one vertex from each triangle in a variable choice gadget and exactly one variable selection vertex per variable set gadget. Note that we cannot replace a variable selection vertex by a vertex marked $z_{1}$ or $z_{2}$ : either it is already dominated, or we also must choose its twin, but we do not have enough vertices for this.

From each triangle in a variable choice gadget, we must choose either the vertex marked 0 or 1 , since otherwise one of the variable selection vertices will not be dominated. This dominates all but one of the variable selection vertices, which must be the variable selection vertex that is in the dominating set. We claim that we satisfy the formula by setting exactly these variables to true and all others to false. (Note also that we set exactly one variable per set $X_{i, j}$ to true this way.) Indeed, the clause vertex needs to be dominated by one of the variable selection vertices, and by definition this implies that the corresponding chosen variable satisfies the clause.

The path decomposition can be constructed as follows. Let $V_{i}$ for $i \in[1, r-1]$ be the set of all vertices in the variable choice gadgets of all sets $X_{i, j}$ and $X_{i+1, j}$ with $j \in[1, k]$. For any given $i$, we create a path decomposition that covers all vertices of $V_{i}$ and all clause gadgets connected to $V_{i}$. We do this by sorting the clauses in any order, and then one by one traversing the clause checking gadgets. Each time, we begin by adding the $z$-vertices and the clause vertex of the gadget to the current bag. Then, iteratively we add and remove all the 
variable representing vertices one by one. After this, we remove the $z$-vertices and clause vertex from the bag and continue to the next clause. At any point, there are at most $6 k \cdot t+4 k+2$ vertices in a bag: $6 k \cdot t$ from the set $V_{i}, 4 k$ $z$-variables, one clause vertex and one variable representing vertex. We then continue the path decomposition by removing all vertices related to variable choice gadgets of the set $X_{i}$ and adding all vertices related to variable choice gadgets of the set $X_{i+2}$.

As the construction can be executed in logarithmic space, the result follows.

\subsection{Scheduling with precedence constraints}

In 1995, Bodlaender and Fellows [8] showed that the problem to schedule a number of jobs of unit length with precedence constraints on $K$ machines, minimizing the makespan, is $W[2]$-hard, with the number of machines as parameter. A closer inspection of their proof shows that $W[2]$-hardness also applies when we take the number of machines and the width of the partial order as combined parameter. In this subsection, we strengthen this result, showing that the problem is XNLP-complete (and thus also hard for all classes $W[t], t \in \mathbf{Z}^{+}$.) In the notation used in scheduling literature to characterize scheduling problems, the problem is known as $P \mid$ prec, $p_{j}=1 \mid C_{\max }$, or, equivalently, $P \mid$ prec, $p_{j}=p \mid C_{\max }$.

Scheduling with Precedence Constraints

Given: $K, D$ positive integers; $T$ set of tasks; $\prec$ partial order on $T$ of width $w$.

Parameter: $K+w$.

Question: Is there a schedule $f: T \rightarrow[1, D]$ with $\left|f^{-1}\{i\}\right| \leq K$ for all $i \in[1, D]$ such that $t \prec t^{\prime}$ implies $f(t)<f\left(t^{\prime}\right)$.

In other words, we parametrise $P \mid$ prec, $p_{j}=1 \mid C_{\max }$ by the number of machines and the width of the partial order.

Theorem 4.6. Scheduling with Precedence Constraints is XNLPcomplete.

Proof. To see that the problem is in XNLP, we define $f^{-1}\{1\}, f^{-1}\{2\}, \ldots$, $f^{-1}\{D\}$ in order as follows. For $i \in[0, D-1]$, we temporarily store a set $S_{i} \subseteq T$ containing the maximal elements of $f^{-1}[1, i]$, initialising $S_{0}=\emptyset$. Each such set $S_{i}$ forms an antichain and hence has size at most $w$, the width of the partial order on $T$. Once $S_{i-1}$ is defined for some $i \in[1, D]$, we define $f^{-1}\{i\}$ by selecting up to $K$ tasks to schedule next, taking a subset of the minimal elements of the set of 'remaining tasks'

$$
R_{i}=\left\{t \in T \mid t \nprec s \text { for all } s \in S_{i-1}\right\} .
$$

By definition, for $t \in R_{i}$, we find that $t \nprec s$ for all $s \in f^{-1}[1, i-1]$. In order to update $S_{i}$ given $S_{i-1}$, we add all selected elements (which must be maximal) and remove the elements $s \in S_{i-1}$ for which $s \prec t$ for some $t \in f^{-1}\{i\}$. After 
computing $S_{i}$ and outputting $f^{-1}\{i\}$, we remove $S_{i-1}$ and $f^{-1}\{i\}$ from our memory. At any point in time, we store at most $O((K+w) \log |T|)$ bits.

To prove hardness, we transform from the ACCEPTING NNCCM problem, with $k$ given counters with values in $[0, n]$ and set of checks $\left(s_{1}, \ldots, s_{r}\right)$. We adapt the proof and notation of Bodlaender and Fellows 8 .

We create $K=2 k+1$ machines and a set of tasks $T$ with a poset structure on this of width at most $3(k+1)$. Let $c=(k n+1)(n+1)$. The deadline is set to be $D=c r+n+1$. We will construct one sequence of tasks for each of the $k$ counters, with $k n+1$ repetitions of $n+1$ time slots for each of $s_{1}, \ldots, s_{r}$ (one for each value in $[0, n]$ that it might like to check), and then $n$ extra time slots for 'increasing' counters. Each $t \in[1, D]$ can be written in the form

$$
t=(j-1) c+\alpha(n+1)+y
$$

for some $y \in[1, n+1], j \in[1, r], \alpha \in[0, k n]$. We define $j(t)$ and $\alpha(t)$ to be the unique values of $j$ and $\alpha$ respectively for which this is possible. Our construction has the following components.

- Time sequence. We create a sequence $a_{1} \prec a_{2} \prec \ldots \prec a_{D}$ of tasks that represents the time line.

- Time indicators. By adding a task $b$ with $a_{t-1} \prec b \prec a_{t+1}$, we ensure that at time $i$ one less machine is available. We place $k-1$ such tasks at special indicator times. That is, we set

$$
I=\{(j-1) c+\alpha(n+1)+n+1 \mid j \in[1, r], \alpha \in[0, k n]\}
$$

and create a task $b_{t}^{(x)}$ with $a_{t-1} \prec b_{t}^{(x)} \prec a_{t+1}$ for each $x \in[1, k-1]$ and $t \in I$.

- Counter sequences. For each $i \in[k]$, we create a sequence $c_{1}^{(i)} \prec c_{2}^{(i)} \prec$ $\ldots \prec c_{D-n}^{(i)}$ of tasks. If $c_{t-\ell}^{(i)}$ is planned in at the same moment as time vertex $a_{t}$ (for some $\ell \in[0, n]$ and $t \in[n+1, D]$ ) then this is interpreted as counter $i$ taking the value $\ell$ at that time.

- Check tasks. Let $t=(j-1) c+\alpha(n+1)+n+1 \in I$ be the indicator time for $j \in[1, r]$ and $\alpha \in[0, k n]$. If $s_{j}=\left(i_{1}, i_{2}, r_{1}, r_{2}\right)$, then for $x \in\{1,2\}$, we add a check task $d_{t-r_{x}}^{\left(i_{x}\right)}$ 'parallel' to $c_{t-r_{x}}^{\left(i_{x}\right)}$, that is, we add the precedence constraints

$$
c_{t-r_{x}-1}^{\left(i_{x}\right)} \prec d_{t-r_{x}}^{\left(i_{x}\right)} \prec c_{t-r_{x}+1}^{\left(i_{x}\right)} .
$$

Intuitively, the repetitions allow us to assume that concurrent with each time task are exactly $k$ tasks of the form $c_{y}^{(i)}$. For most $t \in[1, D]$, we are happy for a potential $d_{y}^{(i)}$ to be scheduled simultaneously (since we have $2 k$ machines available). However, when $t \in I$ then we only have access to $k+1$ machines, so we may have at most one $d_{y}^{(i)}$ at this time; if $t$ 'corresponds' to $s_{j}=\left(i_{1}, i_{2}, r_{1}, r_{2}\right)$, then only $d_{t-r_{1}}^{\left(i_{1}\right)}, d_{t-r_{2}}^{\left(i_{2}\right)}$ can potentially be planned simultaneously with $a_{t}$, which 


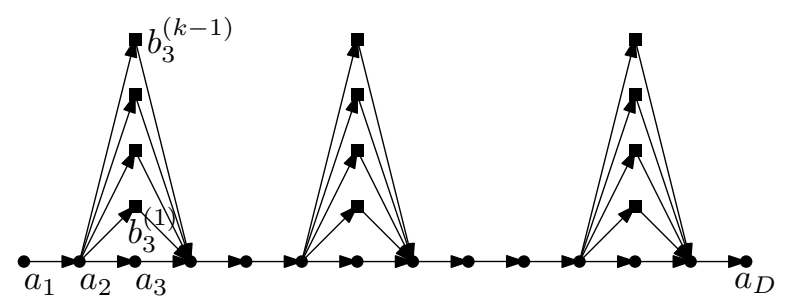

Figure 3: Illustration for a time sequence with time indicators. E.g., in the example, $3 \in I$.

happens exactly if $s_{j}$ rejects (due to the $i_{x}$ th counter being at position $r_{x}$ for both $x \in\{1,2\})$.

The width $w$ of the defined partial order is at most $3(k+1)$. We now claim that there is a schedule if and only if the given machine accepts.

Suppose that the machine accepts. Let $\left(p_{1}, \ldots, p_{r}\right)$ with $p_{j}=\left(p_{j}^{(1)}, \ldots, p_{j}^{(k)}\right) \in$ $[0, n]^{k}$ be the positions of the $k$ counters before $s_{j}$ is checked, for $j \in[1, r]$. We schedule for $t \in[1, D], x \in[1, k-1], i \in[1, k]$, when defined,

$$
\begin{aligned}
& f\left(a_{t}\right)=t, \\
& f\left(b_{t}^{x}\right)=t, \\
& f\left(c_{t}^{(i)}\right)=t+p_{j(t)}^{(i)}, \\
& f\left(d_{t}^{(i)}\right)=t+p_{j(t)}^{(i)} .
\end{aligned}
$$

Recall that $j(t)$ is the unique value $j \in[1, r]$ for which we can write $t=(j-$ $1) c+\alpha(n+1)+y$ for some $\alpha \in[0, k n]$ and $y \in[1, n+1]$. At each $t \in[1, D]$, $f^{-1}\{t\}$ contains exactly one $a_{t}$ and, because the counters are non-decreasing, at most one $c_{t^{\prime}}^{(i)}$ and at most one $d_{t^{\prime}}^{(i)}$ for each $i \in[1, k]$. So when $t \notin I$ (and hence $b_{t}^{x}$ is not defined), $\left|f^{-1}\{t\}\right| \leq 2 k+1$ as desired.

Let $t \in I$ and $j=j(t) \in[1, r], \alpha=\alpha(t) \in[0, k n]$. If $d_{t^{\prime}}^{(i)} \in f^{-1}\{t\}$ for some $i \in[1, k]$, then $t^{\prime}=t-p_{j}^{(i)}$ and $s_{j}=\left(i_{1}, i_{2}, r_{1}, r_{2}\right)$ with $i_{x}=i, r_{x}=p_{j}^{(i)}$ for some $x \in\{1,2\}$. Since the machine accepts, there can be at most one such $i$. Hence $f^{-1}\{t\}$ contains at most one check task when $t \in I$. We again find

$$
\left|f^{-1}\{t\}\right| \leq \underbrace{1}_{a_{t}}+\underbrace{(k-1)}_{b_{t}^{(x)}}+\underbrace{k}_{c_{t^{\prime}}^{(i)}}+\underbrace{1}_{d_{t^{\prime}}^{(i)}} \leq 2 k+1 .
$$

Suppose now that a schedule $f: T \rightarrow[1, D]$ exists. By the precedence constraints, we must have $f\left(a_{t}\right)=t$ for all $t \in[1, D]$ and $f\left(b_{t}^{x}\right)=t$ for all $t \in I$ and $x \in[1, k-1]$. Let $j \in[1, r]$ be given. Let

$$
C=\left\{c_{t}^{(i)} \mid j(t)=j, i \in[1, k]\right\} .
$$

For at least one $\alpha \in[0, k n]$, it must be the case that $\left|f^{-1}\{t\} \cap C\right|=k$ for all $t$ with $\alpha(t)=\alpha$ and $j(t)=j$. This is because for each of the $k$ counter sequences, 
there are at most $n$ places where we may 'skip'; this is why we repeated the same thing $k n+1$ times. We select the smallest such $\alpha$. Note that for all $t \in T$ with $\alpha(t)=\alpha$ and $j(t)=j$, we also find that $f\left(c_{t}^{(i)}\right)=f\left(d_{t}^{(i)}\right)$ for all $i \in[1, k]$.

There is a unique $t \in I$ with $\alpha(t)=\alpha$ and $j(t)=j$; for this choice of $t$, and for all $i \in[1, k]$, we set $p_{j}^{(i)}$ to be the value for which $c_{t-p_{j}^{(i)}}^{(i)} \in f^{-1}\{t\}$. This defines a vector $p_{j} \in[0, n]^{k}$ of positions which we set the counters in before $s_{j}$ is checked. Note that the values of the counters are non-decreasing, and that $s_{j}$ does not fail since $f^{-1}\{t\}$ contains at most one $d_{t^{\prime}}^{(i)}$ (due to its size constraints), which implies that $s_{j}$ accepts.

We remark that it is unclear if the problem is in XNLP when we take only the number of machines as parameter. In fact, it is a longstanding open problem whether Scheduling with Precedence Constraints is NP-hard when there are three machines (see e.g., [26, 40]).

\subsection{Uniform Emulation of Weighted Paths}

In this subsection, we give a proof that the UNIFORM EMULATION OF WEIGHTED PATHS problem is XNLP-complete. The result is a stepping stone for the result that BANDWIDTH is XNLP-complete even for caterpillars with hair length at most three (see the discussion in Subsection 4.5).

The notion of (uniform) emulation of graphs on graphs was originally introduced by Fishburn and Finkel 25] as a model for the simulation of computer networks on smaller computer networks. Bodlaender 3 studied the complexity of determining for a given graph $G$ and path $P_{m}$ if there is a uniform emulation of $G$ on $P_{m}$. In this subsection, we study a weighted variant, and show that already determining whether there is a uniform emulation of a weighted path on a path is hard.

An emulation of a graph $G=(V, E)$ on a graph $H=(W, F)$ is a mapping $f: V \rightarrow W$ such that for all edges $\{v, w\} \in E, f(v)=f(w)$ or $\{f(v), f(w)\} \in$ $F$. We say that an emulation is uniform if there is an integer $c$, such that $\left|f^{-1}\{w\}\right|=|\{v \mid f(v)=w\}|=c$ for all $w \in W$. We call $c$ the emulation factor.

Determining whether there is a uniform emulation of a graph $H$ on a path $P_{m}$ is NP-complete, even for emulation factor 2 , if we allow $H$ to be disconnected. The problem to determine for a given connected graph $H$ if there is a uniform emulation of $H$ on a path $P_{m}$ belongs to XP with the emulation factor $c$ as parameter [3].

Recently, Bodlaender [4] looked at the weighted variant of uniform emulation on paths, for the case that $H$ is a path. Now, we have a path $P_{n}$ and a path $P_{m}$, a weight function $w:[1, n] \rightarrow \mathbf{Z}^{+}$and ask for an emulation $f:[1, n] \rightarrow[1, m]$, such that there is a constant $c$ with $\sum_{i \in f^{-1}\{j\}} w(i)=c$ for all $j \in[1, m]$. Again, we call $c$ the emulation factor.

It is not hard to see that the problem, given $n, m$, and weight function $w:[1, n] \rightarrow \mathbf{Z}^{+}$, to determine if there is a uniform emulation of $P_{n}$ on $P_{m}$ 
with emulation factor $c$ is in XP, with the emulation factor $c$ as parameter; the dynamic programming algorithm from [3] can easily be adapted.

As an intermediate step for a hardness proof for BANDWIDTH, Bodlaender [4] showed that Uniform Emulation of Weighted Paths is hard for all classes $W[t], t \in \mathbf{Z}^{+}$. In the current subsection, we give a stronger result, and show the same problem to be XNLP-complete. Our proof is actually simpler than the proof in 4 - by using ACCEPTING NNCCM as starting problem, we avoid a number of technicalities.

\section{Uniform Emulation of Weighted Paths}

Input: Positive integers $n, m, c$, weight function $w:[1, n] \rightarrow[1, c]$.

Parameter: $c$.

Question: Is there a function $f:[1, n] \rightarrow[1, m]$, such that $f$ is a uniform emulation of $P_{n}$ on $P_{m}$ with emulation factor $c$, i.e., $|f(i)-f(i+1)| \leq 1$ for all $i \in[1, n-1]$ and $\sum_{i \in f^{-1}\{j\}} w(i)=c$ for all $j \in[1, m]$ ?

Theorem 4.7. Uniform Emulation of Weighted Paths is XNLP-complete.

Proof. We first briefly sketch a variant of the dynamic programming algorithm (see [3) that shows membership in XNLP. For $i$ from 1 to $m$, guess which vertices are mapped to $i$. Keep this set, and the sets for $i-1$ and $i-2$ in memory. We reject when neighbors of vertices mapped to $i-1$ are not mapped to $\{i-2, i-1, i\}$ (or not to $\{1,2\}$ or $\{m-1, m\}$ for $i=1$ or $i=m$ respectively) or when the total weight of vertices mapped to $i$ does not equal $c$. We also check whether $\mathrm{cm}=\sum_{i=1}^{n} w(i)$. Since $P_{n}$ is connected, and $f(i)$ is defined for some $i \in[1, n]$, we find that $f(i)$ is defined for all $i \in[1, n]$ (since our check ensures that $f(j)$ is defined for each neighbor $j$ of $i$ in $P_{n}$, and then also for all neighbors of $j$ etcetera). We have $O(c)$ vertices from $P_{n}$ in memory, and thus $O(c \log n)$ bits.

To show that the problem is XNLP-hard, we use a transformation from ACCEPTING NNCCM. Suppose that we are given an $\operatorname{NNCCM}(k, n, s)$, with $s$ a sequence of $r$ checks. We build an instance of UNIFORM EMULATion of Weighted Paths as follows.

First, we define a number of constants:

- $d_{1}=3 k+2$,

- $d_{2}=k \cdot d_{1}+1$,

- $d_{3}=k \cdot d_{2}+1$,

- $c=2 k \cdot d_{3}+1$,

- $n_{0}=3 n+1$,

- $M=1+(r+1) \cdot n_{0}$. 
We construct a weighted path $P_{N}$ for some value $N$ whose value follows from our construction below, and ask for a uniform emulation of $P_{N}$ to $P_{M}$, with emulation factor $c$.

The path $P_{N}$ has three subpaths with different functions. We concatenate these in order to obtain $P_{N}$.

- The floor. We ensure that the $i$ th vertex of the floor must be mapped to the $i$ th vertex of $P_{M}$. We will use this to be able to assume that the counter components always 'run from left to right'.

- The $k$ counter components. Each counter component models the values for one of the counters from the NNCCM and the goal is to ensure the emulation of this part is possible if and only if all the checks succeed.

- The filler path. This is a technical addition aimed to ensure that a total weight of $c$ gets mapped to $j$ for all $j \in[1, M]$.

The floor consists of the following $M$ (weighted) vertices.

- A vertex of weight $c-k \cdot d_{2}$.

- A path with $M-2$ vertices. The $i$ th vertex of $P_{N}$ (and hence the $(i-1)$ th of this part) has weight $c-2 d_{1}+1$ if $i=n_{0} \cdot j+1$ for some $j$ and weight $c-3 d_{1}$ otherwise. The positions of the higher weight of $c-2 d_{1}+1$ are called test positions.

- A vertex of weight $c-k \cdot d_{3}-1$.

We have $k$ counter components, that give after the floor, the successive parts of the 'large path'. The $q$ th counter component has the following successive parts.

- We start with $M-2$ vertices of weight 1.

- We then have a vertex of weight $d_{2}$; this vertex is called the left turning point.

- Then we have $M-2+n$ vertices of weight either 1 or $d_{1}$. The $i$ th vertex has weight $d_{1}$ if and only if $i=n_{0} \cdot j+\alpha$ and the check $s_{j}$ verifies whether counter $q$ is equal to $\alpha$. The $i$ th vertex has weight 1 otherwise. We call this the main path of the counter component.

- A vertex of weight $d_{3}$. This vertex is called the right turning point of the counter component.

Assuming the left and right turning points are mapped to 1 and $M$, the main path of the gadget will be mapped between 2 and $M-1$. These main paths will tell us what the values of the counters are at any moment. The number of shifts then stands for the number a counter gadget represents, i.e. if the $i$ th vertex of the main path is mapped to $i-\alpha+1$, then $\alpha$ is the value of the counter at that 
moment. In this way, assuming that left and right turning points are mapped to 1 and $M$, the value of counters can only increase.

We add a 'filler path' to ensure the total weight of all vertices of $P_{M}$ is at least $M c$, by adding a path of $\min \{\gamma-M c, 0\}$ vertices of weight 1 .

Claim 4.8. The NNCCM described by $(k, n, s)$ accepts if and only if $P_{N}$ with the defined vertex weights has a uniform emulation on $P_{M}$,

Proof. If the NNCCM accepts, then we can build a uniform emulation as follows. Fix some accepting run and let $q(j)$ be the value of counter $q$ at the $j$ th check in this run. Map the $i$ th vertex of the floor to $i$.

Now, successively map each counter component as follows. We start by 'moving back to 1', i.e., we map the $M-2$ vertices of weight 1 to $M-1, M-2$, $\ldots, 2$ and map the left turning point to 1 . To map the vertices on the main path of this counter component, we use the following procedure. For convenience, we define $q(r+1)=n$.

- Set $i=1, p=2$. We see $p \in[1, M]$ as a position on $P_{M}$, and $i \in[1, M+n]$ as the number of the vertex from the main path we are currently looking at. The number $i-(p-1)$ represents the value of the counter.

- Set $j$ to be the smallest integer with $p \leq n_{0} \cdot j+1$; if $j \leq r$, then $n_{0} \cdot j+1$ is the first test position (defined in the floor gadget) after $p$.

- If $i-(p-1)<q(j)$, and $p$ is in the interval $\left[n_{0} \cdot(j-1)+1+n+1, n_{0} \cdot j-n\right]$, then we map both the $i$ th and the $(i+1)$ th vertex of the main path to $p$. We increase $i$ by 2 and $p$ by 1 ; the increase of $i-(p-1)$ by one represents the increase of the counter by one. Otherwise, we map the $i$ th vertex of the main path to $p$, and increase both $i$ and $p$ by one.

Since $q(r+1)=n$, in the end we will map $M-2+n$ vertices to $M-2$ positions.

Note that the final step above does not decrease the value of $i-(p-1)$. The interval $\left[n_{0} \cdot(j-1)+1+n+1, n_{0} \cdot j-n\right]$ contains at least $n \geq q(j)$ integers, and thus we may map two vertices to the same position until we obtain $i-(p-1) \geq q(j)$. We then stop increasing the value $i-p+1$, so $i-p+1=q(j)$ at the $j$ th test position $p=n_{0} \cdot j+1$, and map a single vertex to this.

What vertex gets mapped to the $j$ th test position? If the $q$ th counter does not participate in the $j$ th check, then the vertex will have weight one. Otherwise, suppose that check $s_{j}$ verifies whether counter $q$ is equal to the value $\alpha$. The vertex $i$ mapped to the $j$ th test position has weight $d_{1}$ if and only if $i=n_{0} \cdot j+\alpha$, which is equivalent to $q(j)=\alpha$ since $i-(p-1)=q(j)$ and $p=n_{0} \cdot j+1$. As we were considering an accepting run for the NNCCM, there is at most one counter component that maps a vertex of weight $d_{1}$ (rather than 1 ) to the $j$ th test position.

After we have placed the main path, we send the right turning point to $M$ and continue with the next counter component or, if $q=k$, the filler path. At this point, 1 and $M$ have received weight exactly $c$ (from one floor vertex and $k$ turning points). For all test positions, we have a floor vertex of weight 
$c-2 d_{1}+1$, at most one heavy vertex of weight $d_{1}$, and at most $2 k$ vertices of weight one (at most two per counter component). For a vertex on $P_{M}$ that is not $1, M$ or a test position, we have a floor vertex of weight $c-3 d_{1}$, at most two heavy vertices of weight $d_{1}$ and at most $3 k$ vertices of weight one (per counter component, it obtains at most two vertices for the main path and one for path going left). In all cases, we have a weight less than $c$.

We now use the filler path to make the total weight equal to $c$ everywhere. (By definition, the filler path has enough weight 1 vertices available for this.)

Conversely, suppose that we have a uniform emulation $f$. We cannot map two floor vertices to the same vertex as each has a weight larger than $c / 2$. As we have $M$ floor vertices, one of the following two statements holds:

1. For all $i$, the $i$ th floor vertex is mapped to $i$.

2. For all $i$, the $i$ th floor vertex is mapped to $M-i+1$.

Without loss of generality, we assume that the $i$ th floor vertex is mapped to $i$.

We first show that each right turning point is mapped to $M$ and each left turning point is mapped to 1 . Since $M$ is the only vertex where the available weight (which is at most $c$ minus the weight of the floor vertex) is at least the weight of a right turning point, all right turning points must be mapped to $M$. After this, there is no more weight available on this vertex, and the only vertex where a left turning point can fit is 1 .

We now use the test positions to define values for the counters. Because the main path of the counter component is from a left turning point (mapped to 1) to a right turning point (mapped to $M$ ), at least one vertex of the main path is mapped to the $j$ th test position $n_{0} \cdot j+1$. If the $i$ th vertex of the main path is mapped to the test position, then we set $q(j)=i-n_{0} \cdot j$. (If multiple vertices are mapped to the test position, we choose arbitrarily one of those values.) We find that the values we defined for the counters are non-decreasing since the difference between $i=i(j)$ and $n_{0} \cdot j$ can only become larger for larger $j$.

We are left to prove that this defines an accepting sequence. Assume towards a contradiction that the check $s_{j}=\left(q, q^{\prime}, \alpha_{q}, \alpha_{q^{\prime}}\right)$ fails because of $q(j)=\alpha_{q}$ and $q^{\prime}(j)=\alpha_{q^{\prime}}$. That would imply both the $\left(n_{0} \cdot j+q(j)\right)$ th vertex from the $q$ th counter component and the $\left(n_{0} \cdot j+q^{\prime}(j)\right)$ vertex from the $q^{\prime}$ th counter component were mapped to test position $n_{0} \cdot j+1$. Both these vertices have weight $d_{1}$, by the construction of the main path of counter components. Since we already have a total weight of $c-2 d_{1}+1$ from the floor gadget, the total weight mapped to the $j$ th test position is $>c$. This is a contradiction. Hence the given solution for the NNCCM instance is a valid one.

Theorem 4.7 now follows from Claim 4.8 and observing the resources (logarithmic space, fpt-time) by the transformation.

\subsection{Bandwidth}

In this subsection, we discuss the XNLP-completeness problem of the BANDWIDTH problem. The question where the parameterized complexity of BAND- 
WIDTH lies was actually the starting point for the investigations whose outcome is reported in this paper; with the main result of this subsection (Corollary 4.10) we answer a question that was asked over a quarter of a century ago.

In the BANDWIDTH problem, we are given a graph $G=(V, E)$ and an integer $k$ and ask if there is a bijection $f: V \rightarrow[1,|V|]$ such that for all $\{v, w\} \in E$ : $|f(v)-f(w)| \leq k$. The problem models the question to permute rows and columns of a symmetric matrix, such that all non-zero entries are at a small 'band' along the main diagonal. Already in 1976, the problem was shown to be NP-complete by Papadimitriou [37. Later, several special cases were shown to be hard; these include caterpillars with hairs of length at most three [35]. A caterpillar is a tree where all vertices of degree at least three are on a common path; the hairs are the paths attached to this main path.

We are interested in the parameterized variant of the problem, where the target bandwidth is the parameter:

\section{BANDWIDTH}

Given: Integer $k$, undirected graph $G=(V, E)$

Parameter: $k$

Question: Is there a bijection $f: V \rightarrow[1,|V|]$ such that for all edges $\{v, w\} \in E:|f(v)-f(w)| \leq k$ ?

BANDWIDTh belongs to XP. In 1980, Saxe 41] showed that BANDWIDTH can be solved in $O\left(n^{k+1}\right)$ time; this was later improved to $O\left(n^{k}\right)$ by Gurari and Sudborough [27. In 1994, Bodlaender and et. 9] reported that BANDwIDTH for trees is $W[t]$-hard for all $t \in \mathbf{Z}^{+}$— the proof of that fact was published 26 years later [4. A sketch of the proof appears in the monograph by Downey and Fellows [17. More recently, Dregi and Lokshtanov 19$]$ showed that BANDwIDTH is $W[1]$-hard for trees of pathwidth at most two. In addition, they showed that there is no algorithm for BANDWIDTH on trees of pathwidth at most two with running time of the form $f(k) n^{o(k)}$ assuming the Exponential Time Hypothesis. Recently, Bodlaender 4 published a proof that BANDwIDTH is $W[t]$-hard for all $t$, even for caterpillars with hairs of length at most three. That result is obtained by first showing that Uniform Emulation of Weighted Paths is $W[t]$ hard for all $t$, and then giving a transformation from that problem to BANDWIDTH for caterpillars with maximum hair length tree. The latter transformation uses gadgets from the NP-completeness proof by Monien [35].

\section{Lemma 4.9. BANDWIDTH is in XNLP.}

Proof. This can be seen in different ways: one can look at the XP algorithms for BANDWIDTH from [41] or [27, and observe that when instead of making full tables in the dynamic programming algorithm, we guess from each table one entry, one obtains an algorithm in XNLP.

Alternatively, we loop over all connected components $W$ of $G$, compute its size and for each, guess for $i$ from 1 to $|W|$ the $i$ th vertex in the linear ordering, i.e., $f^{-1}(i)$. Keep the last $2 k+1$ guessed vertices in memory, and verify that all neighbors of $f^{-1}(i-k)$ belong to $f^{-1}[i-2 k, i]$. 
The main result of this subsection is just a corollary of earlier results.

Corollary 4.10. BANDWIDTH for caterpillars with hairs of length at most three is XNLP-complete.

Proof. Membership was argued above in Lemma 4.9 .

Hardness follows directly from the hardness of Uniform EMULATion of Weighted Paths (Theorem 4.7), and the transformation in 4 from Uniform Emulation of Weighted PATHS to BANDWIDTh for caterpillars with hairs of length at most three, as a closer inspection of that proof gives that the reduction is also a pl-reduction.

\subsection{Timed Reconfiguration}

We now consider yet another very different setting: reconfiguration. We first discuss dominating set reconfiguration; in Appendix B, we discuss reconfiguration of cliques and independent sets.

Given a graph $G$ and dominating sets $S$ and $S^{\prime}$, we wish to know whether we can go from one dominating set to the other via a sequence of dominating sets. All dominating sets are of the same size $k$ (which is our parameter) and can be visualised by placing $k$ 'tokens' on the vertices of the graph. The following two rules that are commonly considered for when dominating sets $S_{1}$ and $S_{2}$ are adjacent (that is, can be consecutive in the sequence).

- Token Jumping (TJ). We may 'jump' a single token, that is, the dominating sets $S_{1}$ and $S_{2}$ of size $k$ are adjacent if $S_{1}=S_{2} \backslash\{u\} \cup\{v\}$ for some $u, v \in V(G)$. This rule is equivalent ${ }^{2}$ to Token Addition and Removal (TAR), in which a token can be either added or removed, as long as the total number of tokens does not go above $k+1$.

- Token Sliding (TS). We may 'slide' a single token along an edge, that is, the dominating sets $S_{1}$ and $S_{2}$ of size $k$ are adjacent if $S_{1}=S_{2} \backslash\{u\} \cup\{v\}$ for some $u v \in E(G)$.

The TJ/TAR rule has been most widely studied, and the problem is known to be PSPACE-complete (even for simple graph classes such as planar graphs and classes of bounded bandwidth) [28] and $W[2]$-hard with parameter $k+\ell$, for $k$ the number of tokens and $\ell$ the length of the reconfiguration sequence [36]. Under TS, the problem is also known to be PSPACE-complete even for various restricted graph classes 12 .

It turns out that these problems are XNLP-complete when we bound the number of steps in the reconfiguration sequences as follows.

Timed TS-Dominating Set Reconfiguration

Given: Graph $G=(V, E)$; dominating sets $S, S^{\prime}$ of size $k$; integer $T$ given in unary.

\footnotetext{
${ }^{2}$ Formally, there is a reconfiguration sequence for TJ with $k$ tokens between $S_{1}$ and $S_{2}$ if and only if there is one for TAR with upper bound $k+1$.
} 
Parameter: $k$.

Question: Does there exist a sequence $S=S_{1}, S_{2}, \ldots, S_{T}=S^{\prime}$ of dominating sets of size $k$, with for all $i \in[2, T], S_{i}=S_{i-1} \backslash\{u\} \cup$ $\{v\}$ for some $u v \in E(G)$ ?

Timed TJ-Dominating Set Reconfiguration

Given: Graph $G=(V, E)$; dominating sets $S, S^{\prime}$ of size $k$; integer $T$ given in unary.

Parameter: $k$.

Question: Does there exist a sequence $S=S_{1}, S_{2}, \ldots, S_{T}=S^{\prime}$ of dominating sets of size $k$, with for all $i \in[2, T], S_{i}=S_{i-1} \backslash\{u\} \cup$ $\{v\}$ for some $u, v \in V(G)$ ?

We first consider the token sliding rule and then explain how to adjust the reduction for the token jumping rule.

Theorem 4.11. Timed TS-Dominating Set Reconfiguration is XNLPcomplete.

Proof. The problem is in XNLP follows since we can 'explore' all dominating sets that can be reached from $S$ within $T$ steps nondeterministically, storing the current positions of the $k$ counters and the number of steps taken so far using $O(k \log n)$ bits, accepting if the current position encodes $S^{\prime}$ and halting after $T$ steps.

We prove hardness via a reduction from PARTitioned Regular Chained Weighted Positive CNF-Satisfiability. We obtain $r$ sets of Boolean variables $X_{1}, \ldots, X_{r}$, a Boolean formula $F_{1}$, and a partition of each set $X_{i}$ into sets $X_{i, 1}, \ldots, X_{i, k}$, each of size $q$. We will assume that $r$ is even. We will create a graph and dominating sets $S, S^{\prime}$ of size $2 k+2$ such that $S$ can be reconfigured to $S^{\prime}$ in $T=\frac{5}{2} r-2$ steps if and only if $\wedge_{1 \leq i \leq r-1} F_{1}\left(X_{i}, X_{i+1}\right)$ can be satisfied by setting exactly one variable to true from each set $X_{i, j}$.

The idea of the construction is to create a 'timer' of $2 r-2$ steps, of which each dominating set in the sequence must contain exactly one element, and enforce that the first dominating set $S$ contains the start vertex $t_{0}$ whereas the second dominating set $S^{\prime}$ contains the ending vertex $t_{2 r-3}$. This means the token on $t_{0}$ needs to slide over the timeline, and we use the time constraint to enforce this can happen in a single fashion, using the vertices $t_{2 i}$ to 'move $k$ tokens from $X_{i}$ to $X_{i+2}$ ' and the vertex $t_{2 i-1}$ to 'verify whether the formula $F_{1}\left(X_{i}, X_{i+1}\right)$ holds'.

Our construction has the following parts:

- A 'timer': a path with vertices $t_{0}, t_{1}, \ldots, t_{2 r-3}$, along with two time guardians $g_{t, 1}, g_{t, 2}$ that are adjacent to $t_{i}$ for all $i \in[0,2 r-3]$ and nothing else. In order to dominate the two time guardians using only a single vertex, we need to choose one of the $t_{i}$. This part of the construction is illustrated in Figure 4

- For each variable $x$ a vertex $v_{x}$. 


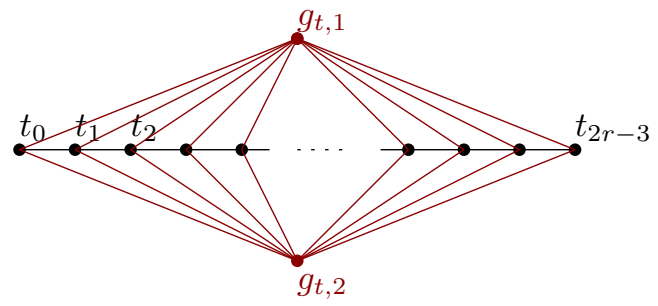

Figure 4: In the time line, at least one vertex needs to get chosen in order to dominate the time guardian vertices $g_{t, 1}$ and $g_{t, 2}$.

- A 'dominator' vertex $d$ with a pendant vertex $p_{d}$ (which is only adjacent to $d$ and enforces that we must always use one token for $d$ or $p_{d}$ ). We use $d$ to dominate all the $t_{i}$ and all the variable vertices.

- For each clause $\phi$ in the Boolean formula $F_{1}$, a vertex $w_{\phi, i}$ for all $i \in$ $[1, r-1]$. The vertex $w_{\phi, i}$ is adjacent to vertex $v_{x}$ for $x \in\left(X_{i} \cup X_{i+1}\right) \cap \phi$ (that is, setting $x$ to true satisfies $\phi$ ). Moreover, $w_{\phi, i}$ is adjacent to all $t_{j}$ with $j \neq 2 i-1$.

- Vertices $m_{i, j}$ for $i \in[1, r], j \in[1, k]$. These will ensure that we move tokens from variables in $X_{i}$ to variables in $X_{i+2}$ at certain time steps.

- Vertices $g_{o e, 0, j, a}$ and $g_{o e, 1, j, a}$ for all $j \in[1, k]$ and $a \in\{1,2\}$. For $a \in\{1,2\}$ and $j \in[1, k]$, the vertex $g_{o e, 0, j, a}$ (respectively $g_{o e, 1, j, a}$ ) is adjacent to all vertices $v_{x}$ for $x \in X_{i, j}$ with $i$ even (respectively odd) and nothing else; these ensure that for each 'variable group' we always have at least one token on a vertex corresponding to this group.

- Start vertices $s_{1}, \ldots, s_{2 k}$ to add to the initial dominating set $S$. The vertices $s_{1}, \ldots, s_{k}$ are adjacent to $v_{x}$ for all $x \in X_{1}$. The vertices $s_{k+1}, \ldots, s_{2 k}$ are adjacent to $v_{x}$ for all $x \in X_{2}$.

- End vertices $s_{1}^{\prime}, \ldots, s_{2 k}^{\prime}$ to add to the final dominating set $S^{\prime}$. The vertices $s_{1}^{\prime}, \ldots, s_{k}^{\prime}$ are adjacent to $v_{x}$ for all $x \in X_{r-1}$. The vertices $s_{k+1}^{\prime}, \ldots, s_{2 k}^{\prime}$ are adjacent to $v_{x}$ for all $x \in X_{r}$.

There are some further edges as explained later. An outline of the construction is depicted in Figure 5 .

Due to the dominator vertex and its pendant vertex, we do not have to worry about dominating the vertices $t_{i}$ and the variable vertices.

The guardian vertices enforce that we need to place a minimum number of tokens on certain vertex sets in order to have a dominating set. One token is always on the dominator vertex; we will call this the dominator token. We need $2 k$ tokens on various variable sets, which we refer to as variable tokens and one token on one of the $t_{i}$, which we refer to as the time token. Since we only have $2 k+2$ tokens, there are no further tokens available and we need to place exactly 


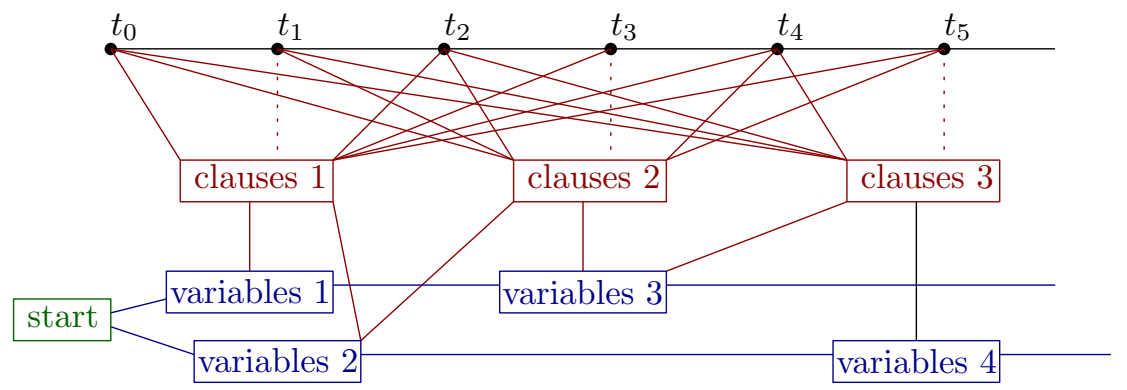

Figure 5: An overview of the construction is given.

these tokens at each time step. In particular, at each time step we can either move the timer token within the timer, or move the variable tokens within their respective sets.

We set $S=\left\{t_{0}, d, s_{1}, \ldots, s_{2 k}\right\}$ and set $S^{\prime}=\left\{t_{2 r-3}, d, s_{1}^{\prime}, \ldots, s_{2 k}^{\prime}\right\}$.

For $i \in[1, r-1]$, the clause vertices $w_{\phi, i}$ are non-adjacent to $t_{j}$ if $j \neq 2 i-1$; this ensures that when the time token is at $t_{2 i-1}$, the variable tokens need to take care of dominating $w_{\phi, i}$ (which happens if and only if they are placed in a satisfying assignment).

For $i \in[1, r-2]$, we make $v_{x}$ adjacent to $v_{y}$ for all $x \in X_{i}$ and $y \in X_{i+2}$. This allows us to 'slide' variable tokens from $X_{1}$ to $X_{3}$ to $X_{5}$ etcetera. For $i \in[3, r]$, we ensure that variable tokens are moved from $X_{i-2}$ to $X_{i}$ when the time token is at $t_{2(i-2)}$ using the move vertices $m_{i, \ell}$; these are adjacent to $t_{j}$ for all $j \neq 2(i-2)+1$ and to all $v_{x}$ for $x \in X_{i, \ell}$. For $i \in[1,2]$, the move vertices $m_{i, \ell}$ are adjacent to $v_{x}$ for all $x \in X_{i, \ell}$ and to all $t_{j}$ for $j \neq 1$. Before the time token can be moved from $t_{0}$ to $t_{1}$, the tokens on the start vertices need to be moved from $S$ to the $2 k$ variable sets $X_{i, \ell}$ for $i \in\{1,2\}, \ell \in[1, k]$ in order to dominate the $m_{i, \ell}$.

To move tokens from $S$ to $S^{\prime}$, we need to move the $2 k$ tokens via the variable vertices. This takes at least $r / 2+1$ moves, as each token can go through either the odd or the even variable sets. The token on $t_{0}$ has to move to $t_{2 r-3}$, which takes at least $2 r-3$ moves. We set the time bound to $T=r / 2+1+2 r-3$ so that we are enforced to exactly take these steps. In particular, for each $i \in[1, r]$ and $\ell \in[1, k]$, there is a unique $x \in X_{i, \ell}$ for which a variable token gets placed on $v_{x}$ for some $x \in X_{i, \ell}$ in a valid reconfiguration sequence.

In conclusion, there is a reconfiguration sequence from $S$ to $S^{\prime}$ within $T$ steps if and only if we can select one variable per $X_{i, \ell}$ so that when these are set to true, the formula $\wedge_{1 \leq i \leq r-1} F_{1}\left(X_{i}, X_{i+1}\right)$ is satisfied.

The construction for the token jumping rule is similar, so we only sketch it below.

Theorem 4.12. Timed TJ-Dominating Set Reconfiguration is XNLPcomplete. 


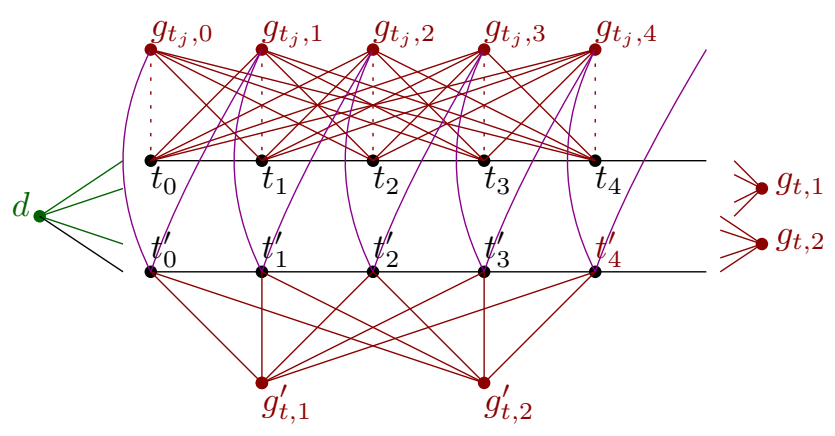

Figure 6: This 'timer' mimicks token sliding under the token jumping rule.

Sketch. We expand on the construction for token sliding as depicted in Figure 6.

We create a second path $t_{0}^{\prime}, \ldots, t_{2 r-3}^{\prime}$. For each $i \in[0,2 r-3]$, we create a guardian vertex $g_{t_{j}, i}$ adjacent to $t_{j}$ for all $j \neq i$ and to $t_{i-1}^{\prime}, t_{i}^{\prime}$ (if these exist).

The $t_{i}^{\prime}$ are also dominated by the dominator vertex $d$, and also have two private guardian vertices $g_{t, 1}^{\prime}, g_{t, 2}^{\prime}$. We will increase the number of tokens by one to $2 r+3$, add $t_{0}^{\prime}$ to $S$ and $t_{2 r-3}^{\prime}$ to $S^{\prime}$ and increase the allowed time $T$ by $2 r-3$ in order to ensure we can exactly slide the second timer token from $t_{0}^{\prime}$ to $t_{2 r-3}^{\prime}$ along the time line.

Suppose we have placed $2 k+3$ tokens in a way that dominates all vertices. Then there is exactly one token on some $t_{i}$ and exactly one on some $t_{i^{\prime}}^{\prime}$. Since $g_{t_{j}, i}$ is also dominated, we find $i^{\prime} \in\{i, i-1\}$. Hence in order to move $t_{0}$ to $t_{2 r-3}$ and $t_{0}^{\prime}$ to $t_{2 r-3}^{\prime}$, we can need to pass through the configurations

$$
\left(t_{0}, t_{0}^{\prime}\right) \rightarrow\left(t_{1}, t_{0}^{\prime}\right) \rightarrow\left(t_{1}, t_{1}^{\prime}\right) \rightarrow\left(t_{2}, t_{1}^{\prime}\right) \rightarrow\left(t_{2}, t_{2}^{\prime}\right) \rightarrow \ldots
$$

and this enables us to use the same construction as for token sliding.

Similar to Timed Dominating Set Reconfiguration, we can define Timed Independent Set Reconfiguration and Timed Clique ReconFiguration. In Appendix B we give a proof that these are XNLP-complete, again assuming the number of steps are given in unary. We remark that many other solution concepts have been studied for reconfiguration, such as satisfiability and coloring versions, and we refer the reader to the survey [45] for more information.

\section{Conclusion}

We end the paper with some discussions and open problems. We start by discussing a conjecture on the space usage of XNLP-hard problems, then discuss the type of reductions we use, and then give a number of open problems. 


\subsection{Space efficiency of XNLP-hard problems}

Pilipczuk and Wrochna [39] made the following conjecture. In the LONGEST Common Subsequence problem, we are given $k$ strings $s^{1}, \ldots, s^{k}$ over an alphabet $\Sigma$ and an integer $r$ and ask if there is a string $t$ of length $r$ that is a subsequence of each $s^{i}, i \in[1, k]$.

Conjecture 5.1 (Pilipczuk and Wrochna [39]). The Longest Common SubSEQUENCE problem has no algorithm that runs in $n^{f(k)}$ time and $f(k) n^{c}$ space, for a computable function $f$ and constant $c$, with $k$ the number of strings, and $n$ the total input size.

Interestingly, this conjecture leads to similar conjectures for a large collection of problems. As Longest Commom SubSEquence with the number of strings $k$ as parameter is XNLP-complete [21, Conjecture 5.1 is equivalent to the following conjecture. Recall that the class XNLP is the same as the class $N[$ fpoly, $f \log n]$.

Conjecture 5.2. $N[$ fpoly, $f \log n] \nsubseteq D\left[n^{f}, f p o l y(n)\right]$.

If Conjecture 5.1 holds, then no XNLP-hard problem has an algorithm that uses XP time and simultaneously 'FPT' space (i.e., space bounded by the product of a computable function of the parameter and a polynomial of the input size). Thus, XNLP-hardness proofs yield conjectures about the space usage of XP algorithms, and Conjecture 5.1 is equivalent to the same conjecture for Bandwidth, List Coloring parameterized by pathwidth, Chained CNFSATisfiability, etc.

\subsection{Reductions}

In this paper, we mainly used parameterized logspace reductions (pl-reductions), i.e., parameterized reductions that run in $f(k)+O(\log n)$ space, with $f$ a computable function.

Elberfeld et al 21] use a stronger form of reductions, namely parameterized first-order reductions or pFO-reductions, where the reduction can be computed by a logarithmic time-uniform para $A C^{O}$-circuit family. In [21], it is shown that Timed Non-Deterministic Cellular Automaton and Longest Common SUBSEQUENCE (with the number of strings as parameter) are XNLP-complete under pFO-reductions. We have chosen to use the easier to handle notion of logspace reductions throughout the paper, and not to distinguish which steps can be done with pl-reductions and which not.

One might want to use the least restricted form of reductions, under which XNLP remains closed, and that are transitive, in order to be able to show hardness for XNLP for as many problems as possible. Instead of using $O(f(k)+$ $\log n)$ space, one may want to use $O(f(k) \cdot \log n)$ space - thus allowing to use

a number of counters and pointers that depends on the parameter, instead of being bounded by a fixed constant. However, it is not clear that XNLP is 
closed under parameterized reductions with a $O(f(k) \cdot \log n)$ space bound, as the reduction may use $O\left(n^{f(k)}\right)$ time.

To remedy this, we can simultaneously bound the time and space of the reduction. A parameterized tractable logspace reduction (ptl-reductions) is a parameterized reduction that simultenously uses $O(f(k) \cdot \log n)$ space and $O(g(k)$. $n^{c}$ ) time, with $f$ and $g$ computable functions, $k$ the parameter, and $n$ the input size. One can observe that the same argument ('repeatedly recomputing input bits when needed') that shows transitivity of L-reductions (see [2, Lemma 4.15]) can be used to show transitivity of parameterized tractable logspace reductions (and of parameterized logspace reductions).

We currently are unaware of a problem where we would use ptl-reductions instead of pl-reductions. However, the situation reminds of a phenomenon that also shows up for hardness proofs for classes in the W-hierarchy. Pl-reductions allow us to use time that grows faster than polynomial in the parameter value. If we have an fpt-reduction that uses $O\left(f(k) n^{c}\right)$ time with $c$ a constant, and $f$ a polynomial function, then this reduction is also a many-to-one reduction, and could be used in an NP-hardness proof for the unparameterized version of the problem. Most but not all fpt-reductions from the literature have such a polynomial time bound. However, in the published hardness proofs, the distinction is usually not made explicit.

\subsection{Candidate XNLP-complete problems}

In this paper, we introduced a new parameterized complexity class, and showed a number of parameterized problems complete for the class, including BANDWIDTH. We expect that there are more problems complete for XNLP. Typical candidates are problems that are known to be hard for $W[t]$ for all integers $t$. Possibly, in some cases, only small modifications of proofs may be needed, but in other cases, new proofs have to be invented. A number of such candidates are the following:

- Linear graph ordering problems, like Colored Cutwidth (and variants), Feasible Register Allocation, Triangulating Colored Graphs (see [10]), Topological Bandwidth.

- Domino Treewidth, see [7]. (We conjecture that XNLP-hardness can be proved with help of ACCEPTING NNCCM; membership in XNLP is unclear due to the tree-like structure of positive instances.)

- Shortest Common Supersequence as mentioned in [17].

- Restricted Completion to a Proper Interval Graph with Bounded Clique Size, see [33,

- Problems parameterized by treewidth or pathwidth that are known to be in XP but not in FPT. Membership in XNLP for several problems parameterized by treewidth is also open (including Dominating SET and 
INDEPENDENT SET). See e.g. [43] for some candidates for problems that might be XNLP-hard when parameterized by treewidth or pathwidth.

\subsection{Complexity theoretic questions}

We end this paper with a few related complexity theoretic open problems.

- Is XNLP closed under complementation? (This is the case for NL but probably not for NP.)

- Is XNLP unequal to XNL, under some well established assumptions?

- Can we get a problem not allowing 'best of both worlds', i.e. that can be solved in either log-space or in fpt time, but not both simultaneously?

\section{References}

[1] A. Abboud, K. Lewi, and R. Williams. Losing weight by gaining edges. In A. S. Schulz and D. Wagner, editors, Algorithms - ESA 2014 - 22th Annual European Symposium, Wroclaw, Poland, September 8-10, 2014. Proceedings, volume 8737 of Lecture Notes in Computer Science, pages 112. Springer, 2014.

[2] S. Arora and B. Barak. Computational Complexity: A Modern Approach. Cambridge University Press, 2007.

[3] H. L. Bodlaender. The complexity of finding uniform emulations on paths and ring networks. Information and Computation, 86(1):87-106, 1990.

[4] H. L. Bodlaender. Parameterized complexity of bandwidth of caterpillars and weighted path emulation. arXiv:2012.01226, 2020. To appear in Proceedings WG 2021.

[5] H. L. Bodlaender, M. Cygan, S. Kratsch, and J. Nederlof. Deterministic single exponential time algorithms for connectivity problems parameterized by treewidth. Inf. Comput., 243:86-111, 2015.

[6] H. L. Bodlaender, R. G. Downey, M. R. Fellows, M. T. Hallett, and H. T. Wareham. Parameterized complexity analysis in computational biology. Comput. Appl. Biosci., 11(1):49-57, 1995.

[7] H. L. Bodlaender and J. Engelfriet. Domino treewidth. J. Algorithms, 24(1):94-123, 1997.

[8] H. L. Bodlaender and M. R. Fellows. $W[2]$-hardness of precedence constrained $K$-processor scheduling. Operations Research Letters, 18(2):93-97, 1995 . 
[9] H. L. Bodlaender, M. R. Fellows, and M. Hallett. Beyond NP-completeness for problems of bounded width: Hardness for the $W$ hierarchy. In Proceedings of the Twenty-Sixth Annual ACM Symposium on Theory of Computing, STOC 1994, pages 449-458, New York, 1994. ACM Press.

[10] H. L. Bodlaender, M. R. Fellows, M. T. Hallett, T. Wareham, and T. J. Warnow. The hardness of perfect phylogeny, feasible register assignment and other problems on thin colored graphs. Theor. Comput. Sci., 244(12):167-188, 2000.

[11] H. L. Bodlaender and R. H. Möhring. The pathwidth and treewidth of cographs. SIAM J. Discret. Math., 6(2):181-188, 1993.

[12] M. Bonamy, P. Dorbec, and P. Ouvrard. Dominating sets reconfiguration under token sliding. arXiv:1912.03127, 2019.

[13] Y. Chen, J. Flum, and M. Grohe. Bounded nondeterminism and alternation in parameterized complexity theory. In 18th Annual IEEE Conference on Computational Complexity (Complexity 2003), 7-10 July 2003, Aarhus, Denmark, pages 13-29. IEEE Computer Society, 2003.

[14] M. Cygan, F. V. Fomin, L. Kowalik, D. Lokshtanov, D. Marx, M. Pilipczuk, M. Pilipczuk, and S. Saurabh. Parameterized Algorithms. Springer, 2015.

[15] R. G. Downey and M. R. Fellows. Fixed-parameter tractability and completeness I: Basic results. SIAM J. Comput., 24(4):873-921, 1995.

[16] R. G. Downey and M. R. Fellows. Fixed-parameter tractability and completeness II: On completeness for $W[1]$. Theoretical Computer Science, 141(1\&2):109-131, 1995.

[17] R. G. Downey and M. R. Fellows. Parameterized Complexity. Springer, 1999.

[18] R. G. Downey and M. R. Fellows. Fundamentals of Parameterized Complexity. Texts in Computer Science. Springer, 2013.

[19] M. S. Dregi and D. Lokshtanov. Parameterized complexity of bandwidth on trees. In J. Esparza, P. Fraigniaud, T. Husfeldt, and E. Koutsoupias, editors, 41st International Colloquium on Automata, Languages, and Programming, ICALP 2014, volume 8572 of Lecture Notes in Computer Science, pages 405-416. Springer, 2014.

[20] M. Elberfeld, A. Jakoby, and T. Tantau. Logspace versions of the theorems of Bodlaender and Courcelle. In 51th Annual IEEE Symposium on Foundations of Computer Science, FOCS 2010, October 23-26, 2010, Las Vegas, Nevada, USA, pages 143-152. IEEE Computer Society, 2010.

[21] M. Elberfeld, C. Stockhusen, and T. Tantau. On the space and circuit complexity of parameterized problems: Classes and completeness. Algorithmica, 71(3):661-701, 2015. 
[22] M. R. Fellows, F. V. Fomin, D. Lokshtanov, F. A. Rosamond, S. Saurabh, S. Szeider, and C. Thomassen. On the complexity of some colorful problems parameterized by treewidth. Inf. Comput., 209(2):143-153, 2011.

[23] M. R. Fellows, D. Hermelin, F. A. Rosamond, and S. Vialette. On the parameterized complexity of multiple-interval graph problems. Theoretical Computer Science, 410(1):53-61, 2009.

[24] M. R. Fellows and F. A. Rosamond. Collaborating with Hans: Some remaining wonderments. In F. V. Fomin, S. Kratsch, and E. J. van Leeuwen, editors, Treewidth, Kernels, and Algorithms - Essays Dedicated to Hans L. Bodlaender on the Occasion of His 60th Birthday, volume 12160 of Lecture Notes in Computer Science, pages 7-17. Springer, 2020.

[25] J. P. Fishburn and R. A. Finkel. Quotient networks. IEEE Trans. Comput., C-31:288-295, 1982.

[26] M. R. Garey and D. S. Johnson. Computers and Intractability: A Guide to the Theory of NP-Completeness. W. H. Freeman, 1979.

[27] E. M. Gurari and I. H. Sudborough. Improved dynamic programming algorithms for bandwidth minimization and the MinCut linear arrangement problem. J. of Algorithms, 5:531-546, 1984.

[28] A. Haddadan, T. Ito, A. E. Mouawad, N. Nishimura, H. Ono, A. Suzuki, and Y. Tebbal. The complexity of dominating set reconfiguration. Theor. Comput. Sci., 651(C):37-49, 2016.

[29] R. A. Hearn and E. Demaine. Pspace-completeness of sliding-block puzzles and other problems through the nondeterministic constraint logic model of computation. Theor. Comput. Sci., 343:72-96, 2005.

[30] T. Ito, E. D. Demaine, N. J. Harvey, C. H. Papadimitriou, M. Sideri, R. Uehara, and Y. Uno. On the complexity of reconfiguration problems. Theoretical Computer Science, 412(12):1054-1065, 2011.

[31] T. Ito, M. Kamiński, H. Ono, A. Suzuki, R. Uehara, and K. Yamanaka. Parameterized complexity of independent set reconfiguration problems. Discrete Applied Mathematics, 283:336-345, 2020.

[32] K. Jansen and P. Scheffler. Generalized coloring for tree-like graphs. Discret. Appl. Math., 75(2):135-155, 1997.

[33] H. Kaplan and R. Shamir. Pathwidth, bandwidth, and completion problems to proper interval graphs with small cliques. SIAM J. Comput., 25(3):540$561,1996$.

[34] D. Lokshtanov, D. Marx, and S. Saurabh. Known algorithms on graphs of bounded treewidth are probably optimal. ACM Trans. Algorithms, 14(2):13:1-13:30, 2018. 
[35] B. Monien. The bandwidth minimization problem for caterpillars with hair length 3 is NP-complete. SIAM Journal on Algebraic and Discrete Methods, 7:505-512, 1986.

[36] A. E. Mouawad, N. Nishimura, V. Raman, N. Simjour, and A. Suzuki. On the parameterized complexity of reconfiguration problems. Algorithmica, 78(1):274-297, 2017.

[37] C. H. Papadimitriou. The NP-completeness of the bandwidth minimization problem. Computing, 16:263-270, 1976.

[38] K. Pietrzak. On the parameterized complexity of the fixed alphabet shortest common supersequence and longest common subsequence problems. $J$. Comput. Syst. Sci., 67(4):757-771, 2003.

[39] M. Pilipczuk and M. Wrochna. On space efficiency of algorithms working on structural decompositions of graphs. ACM Trans. Comput. Theory, 9(4):18:1-18:36, 2018.

[40] D. Prot and O. Bellenguez-Morineau. A survey on how the structure of precedence constraints may change the complexity class of scheduling problems. Journal of Scheduling, 21(1):3-16, 2018.

[41] J. B. Saxe. Dynamic programming algorithms for recognizing smallbandwidth graphs in polynomial time. SIAM J. Algebraic and Discrete Methods, 1:363-369, 1980.

[42] L. J. Stockmeyer. The polynomial-time hierarchy. Theor. Comput. Sci., $3(1): 1-22,1976$.

[43] S. Szeider. Not so easy problems for tree decomposable graphs. arXiv:1107.1177, 2011.

[44] J. A. Telle and A. Proskurowski. Algorithms for vertex partitioning problems on partial $k$-trees. SIAM J. Discret. Math., 10(4):529-550, 1997.

[45] J. van den Heuvel. The complexity of change. arXiv:1312.2816, 2013.

[46] M. Wehar. On the Complexity of Intersection Non-Emptiness Problems. PhD thesis, University at Buffalo, State University of New York, 2016.

\section{A Acyclic Finite State Automata Intersection}

In this section, we briefly discuss the Finite State Automata Intersection problem, and a variant for acyclic automata. In this problem, we are given $k$ deterministic finite state automata on an alphabet $\Sigma$ and ask if there is a string $s \in \Sigma^{*}$ that is accepted by each of the automata.

In the overview of the parameterized complexity of various problems in [17, the problem is mentioned to be hard for all classes $W[t], t \in \mathbf{Z}^{+}$, when either 
parameterized by the number of machines $k$ or by the combination of the number of machines $k$ and the size of the alphabet $\Sigma$; the result is due to Hallett, but has not been published.

More recently, the problem and many variations were studied by Wehar [46]. Amongst others, he showed that Finite State Automata Intersection with the number of machines as parameter is XNL-complete. He also considered the variant where the automata are acyclic.

ACyClic Finite State Automata Intersection

Input: $k$ deterministic finite state automata on an alphabet $\Sigma$ for which the underlying graphs are acyclic (except for self-loops at an accepting or rejecting state).

Parameter: $k$.

Question: Is there a string $s \in \Sigma^{*}$ that is accepted by each of the automata?

Wehar [46, Chapter 5] showed that Acyclic Finite State Automata INTERSECTION is equivalent under LBL-reductions (parameterized reductions that do not change the parameter) to a version of TIMED CNTMC (see Section 2.4 where the given time bound $T$ is linear. The proof technique of Wehar [46] can also be used to show that Acyclic Finite State Automata IntersecTION is XNLP-complete. Instead, we give below a different simple reduction from Longest Common Subsequence, which is XNLP-complete [21.

LONGEST COMmon SUBSEQUENCE

Input: $k$ strings $s^{1}, \ldots, s^{k}$ over an alphabet $\Sigma$; integer $m$.

Parameter: $k$.

Question: Does there exist a string $s \in \Sigma^{*}$ of length at least $m$ that is a subsequence of $s^{i}$ for all $i \in[1, k]$ ?

Theorem A.1. Acyclic Finite State Automata Intersection is XNLPcomplete.

Proof. For XNLP-membership of ACyclic Finite State Automata InterSECTION, the argument from [46, Proposition 5.1] can be used: guess the solution string one character at the time, and keep track of the states of the machines during these guesses. To see this does not take too much time, let $n$ be the largest number of vertices in a graph underlying one of the machines. Since the graphs are acyclic, all machines enter an accepting or rejecting state within $n$ transitions.

The simple transformation from Longest Common Subsequence parameterized by the number of strings $k$ to ACyclic Finite State Automata INTERSECTION is given below.

Suppose that we are given $k$ strings $s^{1}, \ldots, s^{k} \in \Sigma^{*}$ and an integer $m$. We consider $k+1$ automata on alphabet $\Sigma$ : one for each string and one that checks the length of the solution.

The length automaton has $m+1$ states $q_{0}, \ldots, q_{m}$, where $q_{0}$ is the starting state and $q_{m}$ is the accepting state. For each $i \in[0, m-1]$, and each $\sigma \in \Sigma$ we 
have a transition from $q_{i}$ to $q_{i+1}$ labeled with $\sigma$. We also have for each $\sigma \in \Sigma$ a transition (self-loop) from $q_{m}$ to $q_{m}$. The following claim is easy to observe.

Claim A.2. The length automaton accepts a string $s \in \Sigma^{*}$ if and only if the length of $s$ is at least $m$.

For each $i \in[1, k]$, we have a subsequence automaton for string $s^{i}$. Suppose that its length is $\left|s^{i}\right|=t$. The string automaton for $s^{i}$ has the following $t+2$ states: $q_{0}, \ldots, q_{t}, q_{R}$. All states except for the rejecting state $q_{R}$ are accepting states, and $q_{0}$ is the starting state. The automaton for $s^{i}$ has the following transitions. For each $j \in[0, t-1]$ and $\sigma \in \Sigma$, if the substring $s_{j+1}^{i} \cdots s_{t}^{i}$ contains the symbol $\sigma$, then let $s_{j^{\prime}}^{i}$ be the first occurrence of $\sigma$ in this substring, and take a transition from $q_{j}$ to $q_{j^{\prime}}$ labeled with $\sigma$. (Recall that for each state $q$ and each $\sigma \in \Sigma$, if no transition out of $q$ labeled with $\sigma$ was defined in the previous step, then we take a transition from $q$ to $q_{R}$ labeled with $\sigma$.) The name "subsequence automaton' is explained by the following claim.

Claim A.3. The subsequence automaton for string $s^{i}$ accepts a string $s \in \Sigma^{*}$ if and only if $s$ is a subsequence of $s^{i}$.

Proof. Note that after reading a character, the automaton moves to the index of the next occurrence of this character after the current index. So, when we read a subsequence $s$, we are in state $q_{j}$ when $j$ is the smallest integer with $s$ a subsequence of the substring $s_{1}^{i} \ldots s_{j}^{i}$. When there is no such next character, then $s$ is not a subsequence and we move to the rejecting state.

From the claims above, it follows that a string $s \in \Sigma^{*}$ is a subsequence of $s^{1}, \ldots, s^{k}$ of length at least $m$ if and only if $s$ is accepted by both the length automaton and the subsequence automata of $s^{1}, \ldots, s^{k}$. We can compute the automata in $O(f(k)+\log n)$ space, given the strings $s^{1}, \ldots, s^{k}$ and $m$ (with $n$ the number of bits needed to describe these).

It is also not difficult to obtain XNLP-completeness for the restriction to a binary alphabet. First, add dummy symbols to $\Sigma$ to ensure that the size of the alphabet $\Sigma$ is a power of two. Each transition labeled with a dummy symbol leads to the rejecting state $q_{R}$, i.e., we accept the same set of strings. Now, encode each element of $\Sigma$ with a unique string in $\{0,1\}^{\log |\Sigma|}$. For each state $q$ in the automata, replace the outgoing transitions by a complete binary tree of depth $\log |\Sigma|$ with the left branches labeled by 0 and the right branches labeled by 1 . For a leaf of this tree, look at the string $z \in\{0,1\}^{\log |\Sigma|}$ formed by the labels when one follows the path from $q$ to this leaf. This codes a character in $\Sigma$; now let this leaf have a transition to the state reached from $q$ when this character is read. This straightforward transformation gives an automaton that precisely accepts the strings when we replace each character by its code in $\{0,1\}^{\log |\Sigma|}$. If we apply the same transformation to all automata, we obtain an equivalent instance but with $\Sigma=\{0,1\}$. We can conclude the following result.

Corollary A.4. Acyclic Finite State Automata Intersection is XNLPcomplete for automata with a binary alphabet. 


\section{B Reconfiguration}

In Subsection 4.6, we showed that Timed Dominating Set ReconfiguraTION is XNLP-complete. Here, we give a similar result for TimEd IndEPENDENT Set Reconfiguration and Timed Clique Reconfiguration, again assuming that the number of steps is given in unary. The problem descriptions are the same as for Timed Dominating Set Reconfiguration; we spell out the definition for independent set below for the convenience of the reader.

Timed TS-IndePendent Set Reconfiguration

Given: Graph $G=(V, E)$; independent sets $S, S^{\prime}$ of size $k$; integer $T$ given in unary.

Parameter: $k$.

Question: Does there exist a sequence $S=S_{1}, S_{2}, \ldots, S_{T}=S^{\prime}$ of independent sets of size $k$, with for all $i \in[2, T], S_{i}=S_{i-1} \backslash$ $\{u\} \cup\{v\}$ for some $u v \in E(G)$ ?

Timed TJ-Independent Set Reconfiguration

Given: Graph $G=(V, E)$; independent sets $S, S^{\prime}$ of size $k$; integer $T$ given in unary.

Parameter: $k$.

Question: Does there exist a sequence $S=S_{1}, S_{2}, \ldots, S_{T}=S^{\prime}$ of independent sets of size $k$, with for all $i \in[2, T], S_{i}=S_{i-1} \backslash$ $\{u\} \cup\{v\}$ for some $u, v \in V(G)$ ?

Independent set reconfiguration has been widely studied and is known to be PSPACE-complete for both TJ [30] and TS [29], and $W[1]$-hard for TJ when parameterized by the number of tokens [31. We remark that token jumping with $k$ tokens is equivalent to the token addition-removal rule where the solution always needs to contain at least $k-1$ vertices. On the other hand, token sliding and token jumping are not equivalent. If complements cannot be taken efficiently, specific graph classes are studied or the token sliding rule is used, the clique reconfiguration and the independent set reconfiguration could have different complexities.

In this section, we show that both the timed variants of both clique and independent set reconfiguration are XNLP-complete with parameter the number of tokens. The proofs are very similar, and since the idea of the proof is most naturally phrased for Timed TJ-CLIQue REConfiguration, we present it first for that and then deduce the other variants from it.

Theorem B.1. Timed TJ-Clique Reconfiguration is XNLP-complete, with the number of steps given in unary.

Proof. Membership in XNLP is easy: one can guess the sequence of moves, and keep the current positions of the tokens in memory.

For the hardness, we transform from Chained Multicolored Clique. Suppose that we are given an integer $k$ and a graph $G=(V, E)$ with a partition 
of $V$ into sets $V_{1}, \ldots, V_{r}$. Let $f: V \rightarrow[1, k]$ be a given vertex coloring. We look for a chained multicolored clique $W \subseteq V$, that is, for each $i \in[r],\left|W \cap V_{i}\right|=k$, for each $i \in[1, r-1], W \cap\left(V_{i} \cup V_{i+1}\right)$ is a clique, and for each $i \in[1, r]$ and $j \in[1, k], W \cap V_{i}$ contains exactly one vertex of color $j$.

We may assume that for each edge $\{v, w\} \in E$, if $v \in V_{i}$ and $w \in V_{i^{\prime}}$, then $\left|i-i^{\prime}\right| \leq 1$ and if $i=i^{\prime}$, then $f(v) \neq f(w)$. (Edges that do not fulfill these properties will never contribute to a chained multicolored clique, and thus we can remove them. A transducer exists that removes all edges that do not fulfill the property using logarithmic space.)

We build a graph $H$ as follows.

- Start with $G$.

- Add vertex sets $V_{-1}, V_{0}, V_{r+1}, V_{r+2}$, each of size $k$. Extend $f$ as follows: for each color $j \in[1, k]$, and each of the sets $V_{-1}, V_{0}, V_{r+1}, V_{r+2}$, take one vertex of the set and color it with $j$.

- For all $v \in V_{i}$ and $w \in V_{i^{\prime}}, v \neq w$, add an edge only if at least one of the following holds:

$-i \in\{-1,0\}$ and $i^{\prime} \in\{-1,0\}$, i.e. $V_{-1} \cup V_{0}$ is a clique,

$-i=0$ and $i^{\prime}=1$,

$-i \in\{r+1, r+2\}$ and $i^{\prime} \in\{r+1, r+2\}$, i.e. $V_{r+1} \cup V_{r+2}$ is a clique,

$-i=r$ and $i^{\prime}=r+1$,

$-\left|i-i^{\prime}\right|=2$, and $f(v) \neq f(w)$ : a vertex is adjacent to all vertices 'two sets away', except those with the same color.

We reconfigure a clique with $2 k$ vertices. The initial configuration is $V_{-1} \cup V_{0}$ and the final configuration is $V_{r+1} \cup V_{r+2}$.

Claim B.2. Suppose that $G$ has a chained multicolored clique with $k$ colors. Then we can reconfigure $V_{-1} \cup V_{0}$ to $V_{r+1} \cup V_{r+2}$ with $k \cdot(r+2)$ jumping moves in $H$, with each intermediate configuration a clique.

Proof. Let $W$ be the chained multicolored clique with $k$ colors. Write $W^{\prime}=$ $W \cup V_{-1} \cup V_{0} \cup V_{r+1} \cup V_{r+2}$. The starting configuration is $S=V_{-1} \cup V_{0}$.

Take the following sequence: for $i$ from -1 to $r$, for $j$ from 1 to $k$, let $v$ be the vertex in $W^{\prime} \cap V_{i}$ with $f(v)=j$, and $w$ be the vertex in $W^{\prime} \cap V_{i+2}$ with $f(v)=j$ and update $S$ to $S \cup\{w\} \backslash\{v\}$.

It is easy to verify that at each step $S$ is a clique; the total number of moves equals $k \cdot(r+2)$.

Claim B.3. Suppose that we can reconfigure $V_{-1} \cup V_{0}$ to $V_{r+1} \cup V_{r+2}$ with $k \cdot(r+2)$ jumping moves in $H$, with each intermediate configuration a clique. Then $G$ has a chained multicolored clique with $k$ colors. 
Proof. The level of a vertex $v \in V(G)$ is the unique $i \in[1, r]$ for which $v \in V_{i}$. Note that for each edge $\{v, w\}$ in $H$, if $v \in V_{i}$ and $w \in V_{i^{\prime}}$, then $\left|i-i^{\prime}\right| \leq 2$. Thus, each clique can contain vertices of at most three consecutive levels. Also, vertices in the same level with the same color are not adjacent, and vertices two levels apart with the same color are not adjacent. Thus, if $H$ has a clique with $2 k$ vertices, then this clique is a subset of $V_{i} \cup V_{i+1} \cup V_{i+2}$ for some $i \in[-1, r]$, with the property that for each color $j \in[1, k]$, there is one vertex with this color in $V_{i} \cup V_{i+2}$ and one vertex with this color in $V_{i+1}$.

Denote the initial, final and intermediate clique configurations by $S$. To each such set $S$, we associate a value. The potential $\Phi(S)$ is the sum of all levels of the vertices in a clique $S$.

We now argue that each move of a token can increase $\Phi(S)$ by at most two. For any clique $S$, there is an $i$ with $S \subseteq V_{i} \cup V_{i+1} \cup V_{i+2}$ and $S \cap V_{i} \neq \emptyset$. If $\left|S \cap V_{i}\right| \geq 2$, then after a move of one vertex, there still will be at least one vertex in $S \cap V_{i}$. This means that we can only move to vertices in $V_{i^{\prime}}$ with $i^{\prime} \leq i+2$. If $\left|S \cap V_{i}\right|=1$, then suppose $S \cap V_{i}=\{v\}$ with $f(v)=j$. Now, if we move $v$ then we can only move $v$ to a vertex in $V_{i} \cup V_{i+2}$ with color $j$. In both cases, the potential of $S$ increases by at most two.

The initial configuration $S_{0}$ has $\Phi\left(S_{0}\right)=-k$ and the final configuration $S_{k(r+2)}$ has $\Phi(S)=k \cdot(2 r+3)$. Thus, each of the $k \cdot(r+2)$ moves must increase the potential by exactly two. Thus, each move must take a vertex and move it to a vertex exactly two levels later. As each clique configuration spans at most three levels, we have that after each $k$ th move, the configuration spans two levels, and necessarily is a clique with for each color, one vertex from each of these two levels. The collection of these cliques, except the first and last two, forms a chained multicolored clique in $G$.

To finish the hardness proof, we combine the two claims above and observe that $H$ can be constructed in logarithmic space.

Corollary B.4. Timed TJ-Independent Set Reconfiguration and Timed TS-IndePENDENT Set RECONFIGURATION are XNLP-complete.

Proof. Taking the complement of a graph can be done in logarithmic space; thus, the result follows directly from Theorem B.1. noting that we only 'jump' over non-edges in the proof of Theorem B.1, so that in the complement the reconfiguration sequence adheres to the token sliding rules as well.

For the clique variant, a slight alteration to the current construction works.

Corollary B.5. Timed TS-Clique Reconfiguration is XNLP-complete.

Sketch. We follow the proof of Theorem B.1. We build the graph $H$ in a similar fashion, apart from the edges between pairs of sets $\left(V_{i}, V_{i+2}\right)$. For each $i \in$ $[-1, r]$, we place an edge from $v \in V_{i}$ to $w \in V_{i+2}$ if and only if $f(v) \geq f(w)$.

Inductively, one can show that any clique must still span at most three consecutive layers and that each clique contains exactly one vertex of each color from the even and odd levels at any point. Indeed, the vertex of color $k$ in $V_{-1}$ 
can only slide to a vertex of color $k$ in $V_{1}$, and since the vertices of color $k$ in $V_{1}$ form an independent set, this forces the vertex of color $k-1$ in $V_{-1}$ to slide to a vertex of color $k-1$ in $V_{1}$ etcetera.

The same argument can be used for turning a clique into a configuration sequence; however, now it has become important that we slide the vertices in the right order, that is, the first token to be moved from $V_{i}$ to $V_{i+2}$ needs to be the one on a vertex of color 1 (to ensure we remain a clique at any point).

\section{Log pathwidth}

We study the complexity of the independent set and clique analogues of LoGPathwidth Dominating Set of Subsection 4.2. The independent set variant is defined as follows; the clique variant is defined analogously.

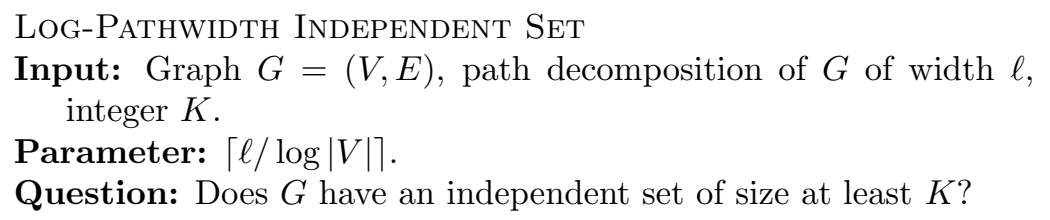

Theorem C.1. Log-Pathwidth Independent Set is XNLP-complete.

Proof. As with Log-Pathwidth Dominating Set, the problem is in XNLP as we can use the standard dynamic programming algorithm on graphs with bounded pathwidth, but guess the table entry for each bag.

We show XNLP-hardness with a reduction from PARITIONED REgUlaR Chained Weighted CNF-Satisfiability. The reduction is similar to the reduction to Log-Pathwidth Dominating Set, but uses different gadgets specific to IndEPENDENT SET. The clause gadget, which will be defined later, is a direct copy of a gadget from [34.

Suppose that $F_{1}, q, X_{1}, \ldots, X_{r}, k, X_{i, j}(1 \leq i \leq r, 1 \leq j \leq k)$ is the given instance of the Paritioned Regular Chained Weighted CNF-SatisfiaBILITY problem. Let $F_{1}=\left\{C_{1}, \ldots, C_{m}\right\}$ and let $\left|C_{i}\right|$ be the number of variables in clause $C_{i}$. We assume $\left|C_{i}\right|$ to be even for all $i \in[1, m]$; if this number is odd, then we can add an additional copy of one of the variables to the clause. Enlarging $\left|X_{i, j}\right|$ by at most a factor of 2 (by adding dummy variables to $X_{i}$ ), we may assume that $t=\log \left|X_{i, j}\right|$ is an integer. (Recall that log has base 2 in this paper.)

Variable choice gadget This gadget consists of $t$ copies of a $K_{2}$ (a single edge), with one vertex marked with a 0 and the other with a 1 . Each element in $X_{i, j}$ can be represented by a unique $q$-bit bitstring. One $K_{2}$ describes one such bit and together these $t$ bits describe one element of $X_{i, j}$, which is going to be the variable that we assume to be true. We will ensure that in a maximal independent set, we need to choose exactly one vertex per copy of $K_{2}$, and hence always represent a variable. 


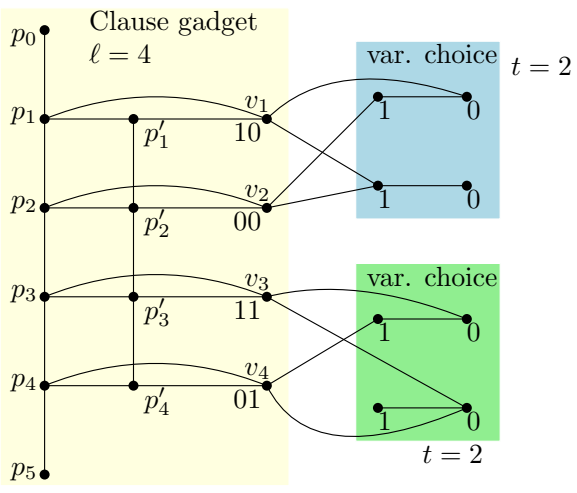

Figure 7: An example of a clause gadget is drawn with two variable gadgets. The vertex $v_{1}$ represents a variable which is encoded by bit string 10 , where $v_{2}$ represents a variable encoded by 00 .

Clause checking gadget The clause checking gadget is a direct copy of a gadget from [34 and is depicted in Figure 7. The construction is as follows. Let $C=x_{1} \vee \cdots \vee x_{\ell}$ be a clause on $X_{i} \cup X_{i+1}$ (for some $i \in[1, r]$ ). We create two paths $p_{0}, \ldots, p_{\ell+1}$ and $p_{1}^{\prime}, \ldots, p_{\ell}^{\prime}$ and add an edge $\left\{p_{j}, p_{j}^{\prime}\right\}$ for $j \in[1, \ell]$. We then add the variable representing vertices $v_{1}, \ldots, v_{\ell}$. For $j \in[1, \ell]$, the vertex $v_{j}$ is connected to $p_{j}$ and $p_{j}^{\prime}$ and to the vertices representing the complement of the respective bits in the representation of $x_{j}$. This ensures that $v_{j}$ can be chosen if and only if the vertices chosen from the variable choice gadget represent $x_{j}$.

For each clause, we add such a clause checking gadget on $3 \ell+2$ new vertices. The pathwidth of this gadget is 4 .

Recall that we assume $\ell$ to be even for all clauses. Each clause checking gadget has the property that it admits an independent set of size $\ell+2$ (which is then maximum) if and only if at least one of the $v$-type vertices is in the independent set, as proved in 34. Otherwise its maximum independent set is of size $\ell+1$. We try to model the clause being satisfied by whether or not the independent set contains $\ell+2$ vertices from this gadget.

The graph $G$ is formed by combining the variable choice and clause checking gadgets.

Claim C.2. There is an independent set in $G$ of size $r k t+(r-1) \sum_{i=1}^{m}\left(\left|C_{i}\right|+2\right)$ if and only if the instance of Partitioned Regular Chained Weighted CNF-SATISFIABILITY has a solution.

Proof. Suppose that there is a valid assignment of the variables that satisfies the clauses. We select the bits that encode the true variables in the variable choice gadgets and put these in the independent set. This forms an independent set because only one element per variable choice gadget is set to true by a valid assignment. As we have $r k$ sets of the form $X_{i, j}$, and each is represented by $t$ 
times two vertices of which we place one in the independent set, we now have $r k t$ vertices in the independent set.

For each clause checking gadget for a clause on $\ell$ variables, we add a further $\ell+2$ vertices to the independent set as follows. Let $x_{i}$ be a variable satisfying the clause. We add the variable representing vertex $v_{i}$ (corresponding to $x_{i}$ ) into the independent set. Since $v_{i}$ is chosen in the independent set, an additional $\ell+1$ vertices can be added from the corresponding clause checking gadget. In total we place $r k t+(r-1) \sum_{i=1}^{m}\left(\left|C_{i}\right|+2\right)$ vertices in the independent set.

To prove the other direction, let $S$ be an independent set of size $r k t+(r-$ 1) $\sum_{i=1}^{m}\left(\left|C_{i}\right|+2\right)$. Any independent set can contain at most $t$ vertices from any variable choice gadget (one per $K_{2}$ ), and at most $|C|+2$ vertices per clause checking gadget corresponding to clause $C$. Hence, $S$ contains exactly $t$ vertices per variable choice gadget and exactly $|C|+2$ vertices per clause gadget.

Consider a variable choice gadget. Since $S$ contains exactly $t$ vertices (one per $K_{2}$ ), a variable from the set $X_{i, j}$ is encoded. It remains to show that we satisfy the formula by setting exactly these variables to true and all others to false. Consider a clause $C$ and its related clause checking gadget. As $|C|+2$ vertices from its gadget are in $S$, this means that at least one of its vertices of the form $v_{i}$ is also in $S$. None of the neighbors of $v_{i}$ can be in $S$, so the corresponding variable $x_{i}$ must be set to true. Thus the clause is indeed satisfied.

A path decomposition can be constructed as follows. For $i=1, \ldots, r$, let $V_{i}$ be the set of all vertices in the variable choice gadgets of the sets $X_{i, j}$ and $X_{i+1, j}$ (for all $j \in[1, k]$ ). We create a sequence of bags that contains $V_{i}$ as well as a constant number of vertices from clause checking gadgets. We transverse the clause checking gadgets in any order. For a given clause checking gadget of a clause on $\ell$ variables, we first create a bag containing $V_{i}$ and $p_{0}, p_{1}, p_{1}^{\prime}$ and $v_{1}$. Then for $s=2, \ldots, \ell$, we add $p_{s}, p_{s}^{\prime}$ and $v_{s}$ to the bag and remove $p_{s-2}, p_{s-2}^{\prime}$ and $v_{s-2}$ (if these exist). At the final step, we remove $p_{\ell-1}, p_{\ell-1}^{\prime}$ and $v_{\ell-1}$ from the bag and add $p_{\ell+1}$. We then continue to the next clause checking gadget. Each bag contains at most $4 k \cdot t$ from the set $V_{i}$ and at most 6 from any clause gadget.

This yields a path decomposition of width at most $4 k t+6 \leq 10 k \log \left|X_{i, j}\right|$ which is of the form $g(k) \log (n)$ for $n$ the number of vertices of $G$ and $g(k)$ a function of the problem we reduced from, as desired. Since $G$ can be constructed in logarithmic space, the result follows.

We now briefly discuss the Log-PATHWIDTh Clique problem. We are given a graph $G=(V, E)$ with a path decomposition of width $\ell$, and integer $K$ and ask if there is a clique in $G$ with at least $K$ vertices. Let $k=\lceil\ell / \log |V|\rceil$.

The problem appears to be significantly easier than the corresponding versions of Dominating Set and Independent Set, mainly because of the property that for each clique $W$, there must be a bag of the path decomposition that contains all vertices of $W$ (see e.g., 11.) Thus, the problem reverts to solving $O(n)$ instances of Clique on graphs with $O(k \log n)$ vertices. This problem is related to a problem called Mini-Clique where the input has a graph with a 
description size that is at most $k \log n$. Mini-Clique is $M[1]$-complete under FPT Turing reductions (see [18, Corollary 29.5.1]), and is a subproblem of our problem, and thus Log-Pathwidth Clique is $M[1]$-hard. However, instances of Log-Pathwidth Clique can have description sizes of $\Omega\left(\log ^{2} n\right)$. The result below shows that it is unlikely that Log-PATHwidth Clique is XNLP-hard.

Proposition C.3. Log-Pathwidth Clique is in $W[2]$.

Proof. Suppose that we are given a graph $G=(V, E)$ and path decomposition $\left(X_{1}, \ldots, X_{r}\right)$ of $G$ of width at most $k \log n-1$. Let $K$ be a given integer for which we want to know whether $G$ has a clique of size $K$. We give a Boolean expression $F$ of polynomial size that can be satisfied with exactly $2 k+2$ variables set to true, if and only if $G$ has a clique of size $K$. (This shows membership in $W[2]$.

First, we add variables $b_{1}, \ldots, b_{r}$ that are used for selecting a bag; we add the clause $\bigvee_{1 \leq i \leq r} b_{i}$.

For each bag $X_{i}$, we build an expression that states that $G\left[X_{i}\right]$ has a clique with $r$ vertices as follows. Partition the vertices of $X_{i}$ in $k$ groups of at most $\log n$ vertices each. For each group $S \subseteq X_{i}$, we have a variable $c_{i, S^{\prime}}$ for each subset $S^{\prime}$ of vertices of the group that induces a clique in $G$. (We thus have at most $k n$ such variables per bag.) The idea is that when $c_{i, S^{\prime}}$ is true, then the vertices in $S^{\prime}$ are part of the clique, and the other vertices in the group are not. The variable $c_{i, S^{\prime}}$ is false if the bag $X_{i}$ is not selected (we add the clause $b_{i} \vee \neg c_{i, S^{\prime}}$ ). For each group $S$, we add a clause that is the disjunction over all cliques $S^{\prime} \subseteq S$ of $c_{i, S^{\prime}}$ and $\neg b_{i}$. This encodes that if the bag $X_{i}$ is selected, then we need to choose a subset $S^{\prime}$ from the group $S$.

With the $b$ - and $c$-variables, we specified a bag and a subset of the vertices of the bag. It remains to add clauses that verify that the total size of all selected subsets equals $K$, and that the union of all selected subsets is a clique. For the latter, we add a clause $\neg c_{i, S^{\prime}} \vee \neg c_{i, S^{\prime \prime}}$ for each pair $S^{\prime}, S^{\prime \prime}$ of subsets of different groups for which $S^{\prime} \cup S^{\prime \prime}$ does not induce a clique. For the former, we number the groups $1,2, \ldots, k$, and create variables $t_{j, q}$ for $j \in[1, k]$ and $q \in[0, K]$. The variable $t_{j, q}$ expresses that we have chosen $q$ vertices in the clique from the first $j$ groups of the chosen bag. For each $j \in[1, k]$, we add the clause $\bigvee_{q} t_{j, q}$. To enforce that the $t$-variables indeed give the correct clique sizes, we add a large (but polynomial) collection of clauses. We add a variable $t_{0,0}$ that must be true (using a one-literal clause). For the $j$ th group with vertex set $S \subseteq X_{i}$, each clique $S^{\prime} \subseteq S$, and each $q, q^{\prime} \in[0, r]$ (with $q=0$ when $j=1$ ), whenever $q+\left|S^{\prime}\right| \neq q^{\prime}$, we have a clause $\neg t_{j-1, q} \vee \neg c_{i, S^{\prime}} \vee \neg t_{j, q^{\prime}}$. Finally, we require (with a one-literal clause) that $t_{k, K}$ holds. If there is a satisfying assignment, then the union of all sets $S^{\prime}$ for which $c_{i, S^{\prime}}$ is true, forms a clique of size $K$ in the graph.

We allow $2 k+2$ variables to be set to true: one variable $b_{i}$ to select a bag, one for $t_{0,0}$, one for a $c_{i, S^{\prime}}$-variable in each of the $k$ groups and one $t_{j, q^{-v} \text {-variable }}$ for each $j \in[1, k]$ (where for $j=k, q=K$ is enforced). 